\title{
Integrated Methodology for the Prediction of Helicopter Rotor Noise at Mission Level
}

\author{
Stavros Vouros ${ }^{\mathrm{a}, *}$, Ioannis Goulos ${ }^{\mathrm{a}}$, Vassilios Pachidis ${ }^{\mathrm{a}}$ \\ ${ }^{a}$ Centre for Propulsion Engineering, School of Aerospace, Transport and Manufacturing, Cranfield University, \\ Bedfordshire, MK430AL, UK \\ * Corresponding author at: Centre for Propulsion Engineering, School of Aerospace, Transport and \\ Manufacturing, Cranfield University, Building 52, GTE Room 5, Bedfordshire, MK430AL, UK, Tel.: +44 (0) \\ 123475 5279,e-mail address: s.vouros@cranfield.ac.uk (S.Vouros)
}

\begin{abstract}
This paper presents an integrated approach for the aeroacoustic assessment of fourdimensional rotorcraft operations. A comprehensive rotorcraft code is utilized to model aircraft flight dynamics across complete missions. A free-wake aero-elastic rotor model is employed to predict high-resolution unsteady airloads, including blade-vortex interactions, at each mission element. A rotor aeroacoustics code is developed to calculate source noise and far-field ground acoustic impact. Time-domain acoustic formulations are used to evaluate near-field noise generation across designated acoustic spherical surfaces surrounding the helicopter main rotor. A numerical procedure is developed for the derivation of acoustic spheres on-the-fly, coupled with trajectory-adaptive ground observer grids. The individual analytical models are incorporated into a mission analysis numerical procedure. The applicability of the integrated method on "real-world" rotorcraft operations is demonstrated for two generic, four-dimensional missions, without the need of pre-stored noise data. The proposed approach provides insight into helicopter noise prediction at mission level, elaborating on the coupling of aeroelastic rotor response with rotorcraft flight dynamics and aeroacoustics.
\end{abstract}

Keywords: Computational aeroacoustics, Helicopter noise prediction, Mission analysis, Acoustic spheres, Ground noise footprint.

\section{Nomenclature}

\section{Roman Symbols}

$A_{c s}$

Blade element cross sectional area

$\mathrm{m}^{2}$

$c_{0}$

Speed of sound of quiescent acoustic medium

$\mathrm{m} / \mathrm{s}$ 
$C_{L} \quad$ Rotor disc rolling moment coefficient $=L /\left(\rho \pi \Omega^{2} R^{5}\right)$

$C_{M} \quad$ Rotor disc pitching coefficient $=M /\left(\rho \pi \Omega^{2} R^{5}\right)$

$C_{n} M^{2} \quad$ Normal force coefficient $=F_{n} /\left(0.5 \rho c_{0}^{2} c_{b}\right)$

$C_{T} \quad$ Rotor disc thrust coefficient $=T /\left(\rho \pi \Omega^{2} R^{4}\right)$

$d \quad$ Perpendicular distance between a point and a vortex filament

$\mathrm{m}$

D Finite difference operator

dy $\quad$ Blade differential span length

$\mathrm{m}$

$f=0 \quad$ Acoustic data line function

$F_{n} \quad$ Sectional normal force

$h$

Flight altitude

$\mathrm{m}$

$H(f) \quad$ Heaviside function

$\vec{l}_{12}$

Vortex filament vector

$\mathrm{m}$

$\vec{L}$

Blade element section loading vector

$\mathrm{N} / \mathrm{m}$

$L$

Rotor rolling moment

$\mathrm{Nm}$

$\vec{M}$

Relative Mach number vector

$|\vec{M}|$

Magnitude of relative Mach number vector

$M$

Rotor pitching moment

$\mathrm{Nm}$

$\hat{n}$

Unit outward normal vector to the source surface, with components $\hat{n}_{j}$

$p$

Local air pressure

$\mathrm{Pa}$

$p_{0}$

Air pressure in the quiescent acoustic medium

$\mathrm{Pa}$

$p^{\prime}$

Acoustic pressure

$\mathrm{Pa}$

$p_{m}^{\prime}$

Monopole acoustic pressure

$\mathrm{Pa}$

$p_{d}^{\prime}$

Dipole acoustic pressure

$\mathrm{Pa}$ 


\begin{tabular}{|c|c|c|}
\hline$P_{i j}$ & Compressive stress tensor & $\mathrm{N} / \mathrm{m}^{2}$ \\
\hline$\vec{r}$ & Position vector of a point on a vortex filament & $\mathrm{m}$ \\
\hline$\hat{r}$ & Unit radiation vector & \\
\hline$r$ & Radiation distance & $\mathrm{m}$ \\
\hline$r_{c}$ & Tip vortex viscous core radius & $\mathrm{m}$ \\
\hline$r_{c 0}$ & Initial tip vortex viscous core radius & $\mathrm{m}$ \\
\hline$\vec{r}_{1}$ & Position vector of a point relative to the beginning of a vortex filament & $\mathrm{m}$ \\
\hline$\vec{r}_{2}$ & Position vector of a point relative to the end of a vortex filament & $\mathrm{m}$ \\
\hline$R(n, m)$ & Shorthand notation function & $\mathrm{m}^{-\mathrm{n}}$ \\
\hline$t$ & Observer time & $\mathrm{s}$ \\
\hline$T$ & Rotor thrust & $\mathrm{N}$ \\
\hline$T_{0}$ & Reference time period & $\mathrm{s}$ \\
\hline$T_{i j}$ & Lighthill stress tensor & $\mathrm{N} / \mathrm{m}^{2}$ \\
\hline$\vec{u}$ & Local fluid velocity, with components $u_{i}$ & $\mathrm{~m} / \mathrm{s}$ \\
\hline$\vec{v}$ & Local velocity of source surface & $\mathrm{m} / \mathrm{s}$ \\
\hline$\vec{V}$ & Velocity vector in rotor wake & $\mathrm{m} / \mathrm{s}$ \\
\hline$\vec{V}_{\text {ind }}$ & Induced velocity vector & $\mathrm{m} / \mathrm{s}$ \\
\hline$V_{\infty}$ & Freestream velocity & $\mathrm{m} / \mathrm{s}$ \\
\hline$W$ & Shorthand notation term & $\mathrm{m} / \mathrm{s}$ \\
\hline$\vec{x}$ & Observer position vector, with components $x_{i}$ & $\mathrm{~m}$ \\
\hline$\vec{y}$ & Source position vector & $\mathrm{m}$ \\
\hline
\end{tabular}

Greek Symbols

$\alpha \quad$ Oseen constant for the vortex core radius

$\alpha_{1} \quad$ Squire parameter for the eddy viscosity factor

$\alpha_{\mathrm{s}} \quad$ Wind tunnel rotor shaft angle $\quad \mathrm{rad}$ 
Dirac delta function

Normalized root-mean-square deviation

\section{Introduction}

Rotorcraft operations are governed by strong variations in flight speed, flight-path angle, altitude and gross weight. These variations are essentially reflected on the acoustic ground footprint throughout the mission-time. Hence, generalized methodologies, capable of resolving the entire flight trajectory are required to address the noise prediction problem from a holistic perspective. Present ground footprint prediction codes mostly rely on source noise databases given in terms of acoustic disturbance over hemispheres surrounding the helicopter. Sound hemispheres are typically provided by either computational or experimental campaigns [1]. The majority of existing mission simulation methods do not directly generate acoustic spheres corresponding to each and every mission element. Instead, two-dimensional interpolation is typically required among a set of available hemispheres to better match the operating conditions of each trajectory waypoint. Acoustic prediction methods based on pre-stored noise hemispheres have been employed within flight procedure impact assessment [2], as well as mission trajectory optimization [3].

A quasi-static acoustic mapping method was introduced[4], [5] to predict blade-vortex interaction noise in maneuvering flight. The term of rotorcraft acceleration parallel to the flight path 
was included in the calculation of rotor tip-path-plane angle. For each waypoint of the maneuvering trajectory, a radiation sphere which corresponded to an equivalent trimmed state was acquired by interpolating among a pre-calculated tabulated set of acoustic spheres. The governing parameters selected for the interpolation were the advance ratio and tip-path-plane angle. Rotor thrust coefficient was not directly included in the set of governing parameters. Greenwood [6] included the rotor thrust coefficient in the set of operating parameters which govern the generation of the acoustic sphere databases. A quasi-static method was developed to predict ground noise impact for short-duration demonstrative maneuvers. The method neglected rotor broadband noise which is a potential source of disturbance for out-of-plane ground observers.

In [7], the GENHEL flight dynamics code was coupled with the PSU-WOPWOP acoustic analogy noise prediction code and applied on low altitude demonstrative maneuvers. After including a free-vortex wake model [8], it has been proven that BVI, when it occurs, is the dominant noise source; however, its prediction comes with high computational expense: up to 50 rotor revolutions were required, even for short-duration maneuvers. Acoustic analogy has also been used for the generation of noise hemispheres, utilizing unsteady free-wake aerodynamics input [9]. The integrated methodology was used for the purposes of noise abatement procedure design, although it was applied to simplified hypothetical flyovers, rather than complete helicopter mission trajectories. Recently, Gennaretti et al. [10], [11] introduced a hemisphere-based approach to predict the acoustic footprint of helicopter unsteady maneuvers. A database of noise spheres was developed, relied on steady flight aeroelastic, aerodynamic and aeroacoustic predictions. The method capabilities have been demonstrated for an indicative approach trajectory.

The acoustic footprint of helicopters has been routinely evaluated for demonstrative, shortrange and short-duration flight procedures. Predictions have been almost entirely based on pre-stored noise hemisphere data-sets, corresponding to a finite number of flight states, representative only of the designated vehicle. However, a "real-world" helicopter mission can have such duration and range that hundreds of square kilometers can be affected for hundreds of minutes time. Along their fourdimensional trajectory, rotorcraft encounter notable variations in flight speed, flight-path angle, altitude, fuel weight and payload [12]. In that context, trim control angles, fuselage attitude angles and 
rotor blade kinematics and loading are continuously varying with time. These fluctuations are directly reflected on the ground noise impact. The applicability of generalized and physics-based methods on the prediction of helicopter rotor noise generation and propagation at mission-level and without any pre-stored noise data has not yet been investigated.

In light of the aforementioned research in the existing literature, the general scope of this work is to develop a generalized and efficient methodology for the prediction of the acoustic footprint of realistic, four-dimensional rotorcraft operations. Specifically, an analytical approach is described that enables the transition from the traditional strategy with pre-stored source noise data-sets to the generation of acoustic spheres on-the-fly resolving the aerodynamics and flight mechanics of each individual flight state. A methodology that employs efficient formulations for the coupled prediction of near-field and far-field rotor noise generation and propagation is developed. A trajectory-adaptive ground observer grid is introduced to reduce the number of observers required and enable the capability to treat long-range and -duration missions. The developed acoustics model is coupled with a mission analysis comprehensive rotorcraft code and a free-wake rotor airloads model. The comprehensive code provides the mission flight dynamics solution to the free-wake rotor airloads model, which calculates the elastic blade kinematics, unsteady loading and blade-vortex interactions (BVIs) necessary for the acoustics prediction. The applicability and robustness of the integrated approach is demonstrated for two generic four-dimensional mission of a twin-engine light (TEL) helicopter model, representative of the MBB Bo 105. The proposed method constitutes a holistic approach for the prediction of helicopter rotor noise at mission level without the need of pre-stored acoustic data.

\section{Methodology}

\subsection{Mission flight dynamics model}

The comprehensive framework "HECTOR" (Helicopter Omni-disciplinary Research Platform) is employed for the calculation of flight kinematics and engine performance across the mission. HECTOR has been extensively described in [12]-[15], therefore only a brief description of the individual modelling methods is provided in this paper. The aeroelastic model for the simulation 
of rotor blade flexibility in the time-domain, is based on the numerical method described in [13]. A minimum potential energy approach, based on Lagrangian kinematics, is employed to obtain the natural vibration characteristics of the main rotor blades for flap-lag-torsion [14]. The structural formulation of the rotor model is coupled with the Leishman-Beddoes unsteady nonlinear blade element aerodynamics model [16], the Peters-He finite state induced flow model [17] and the Goulos dynamic wake curvature model [15]. A globally convergent Newton-Raphson approach is incorporated to obtain the required trim control and fuselage attitude angles for any set of flight conditions utilizing the individual models mentioned above.

Fig. 1 presents the mission analysis numerical procedure incorporated in HECTOR which has been introduced in [12]. The mission path is described in a global geographical system (longitudelatitude) based on the expressions employed by WGS 84 [18]. The overall process involves the breakdown of each designated mission task element into discrete segments with a predefined time step. The helicopter is assumed to be operating in trim and the engine in steady-state off-design during each segment. The initial rotorcraft all-up mass (AUM) is determined based on a user-defined initial guess of the total mission fuel consumption. The helicopter is trimmed depending on the flight conditions defined within the mission. The numerical scheme is applied towards the solution of the Euler's equations of motion for the aircraft fuselage which is treated dynamically as a rigid body with six degrees of freedom, three translational and three rotational.

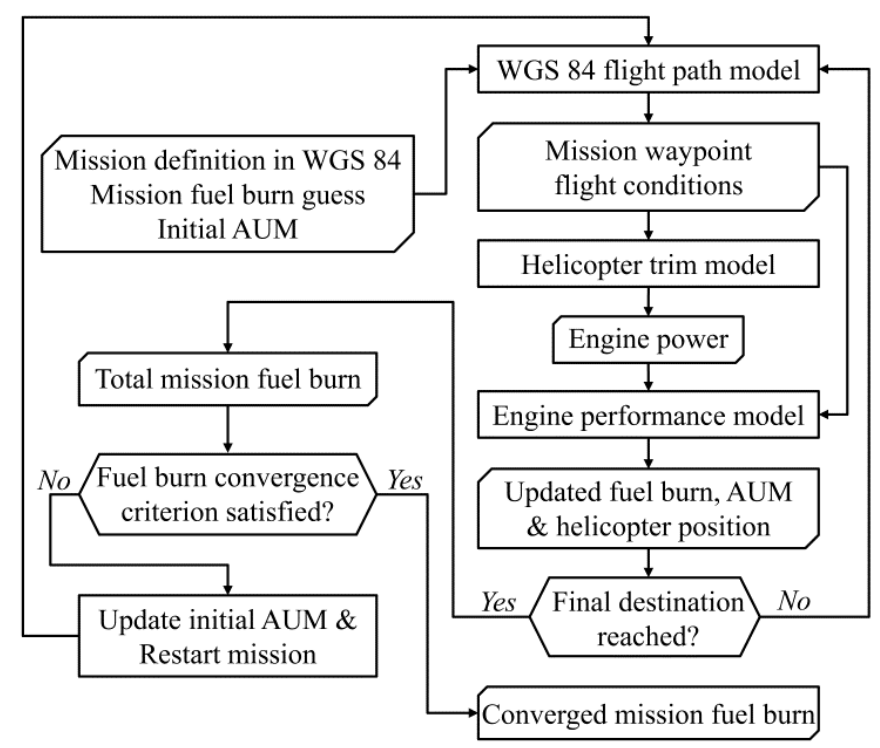

Fig. 1. HECTOR mission analysis numerical procedure. 
The engine performance model (Turbomatch) employed in HECTOR has been developed and improved for over three decades at Cranfield University [19]. Turbomatch is based on zerodimensional analysis of the aero-thermodynamic processes occurring throughout the engine gas path, employing discrete component maps. Therefore, the engine fuel flow is determined among other offdesign performance parameters. The time-dependent fuel consumption is calculated by applying a numerical time integration scheme over the corresponding mission segments. This value is subtracted from the AUM to simulate the helicopter's gradual weight reduction along the mission.

The helicopter's spatial location is updated based on the flight conditions dictated by the flight path model. Having completed a first-pass along the entire mission, the estimated value of total fuel consumption is compared with the initial fuel burn guess. If the obtained percentage difference in mission fuel burn between successive flight path iterations is within a user-defined tolerance, the process is halted. Otherwise, the initial AUM is updated based on the calculated total mission fuel consumption. The overall process is reiterated in a fixed-point manner until the deviation of the calculated fuel consumption from the AUM is below the user-specified convergence criterion.

The procedure described above determines the required rotor thrust $\left(C_{T}\right)$, rolling moment ( $C_{L}$ ) and pitching moment $\left(C_{M}\right)$ coefficients, as well as fuselage attitudes in a computationally inexpensive manner, based on the resolved rotorcraft trim state of each trajectory waypoint. These requirements are then passed to the free-wake aeroelastic rotor model to trim the main rotor and provide the high-resolution airloads necessary for the aeroacoustic predictions.

\subsection{Rotor airloads model}

Rotor induced flowfield is strongly affected by tip vortices which produce high fluctuations to the local blade element flow incidence angles and consequently, rotor airloads. The latter gives rise to high-intensity BVI noise generation. To capture this effect, a wake model which treats discrete flow structures like tip vortices is required. A free-wake, unsteady and aeroelastic rotor model is utilized for this purpose. The rest of individual rotor models are similar to the ones used by HECTOR to maximize consistency. A minimum potential energy method, similar to the one reported in [14], is employed for the estimation of coupled flap-lag-torsion vibration characteristics of the elastic rotor 
blades [20]. The matrix/vector based formulation presented in [13] is employed for modelling the flexible rotor blade kinematics and the Leishman-Beddoes indicial response method [16] for the prediction of unsteady blade airloads. The aforementioned models have been extensively described in the corresponding references; thus further discussion will be omitted.

A relaxation-type free-wake aerodynamics model has been employed for the modelling of unsteady rotor inflow, able to capture the complex blade-vortex interaction phenomena [21], [22]. Each vortex is discretized as a series of straight vortex filaments with Lagrangian markers located at the ends of each filament, as shown in Fig. 2. The governing equation for the convection of the Lagrangian markers is the unsteady Vorticity Transport Equation for incompressible and isotropic fluids:

$\frac{D \vec{\omega}}{D t}=(\vec{\omega} \cdot \nabla) \vec{V}+\nu \nabla^{2} \vec{\omega}$

where $\vec{\omega}$ is the vorticity of the fluid element, $\vec{V}$ is the velocity vector at the same point and $\nu$ is the fluid kinematic viscosity.

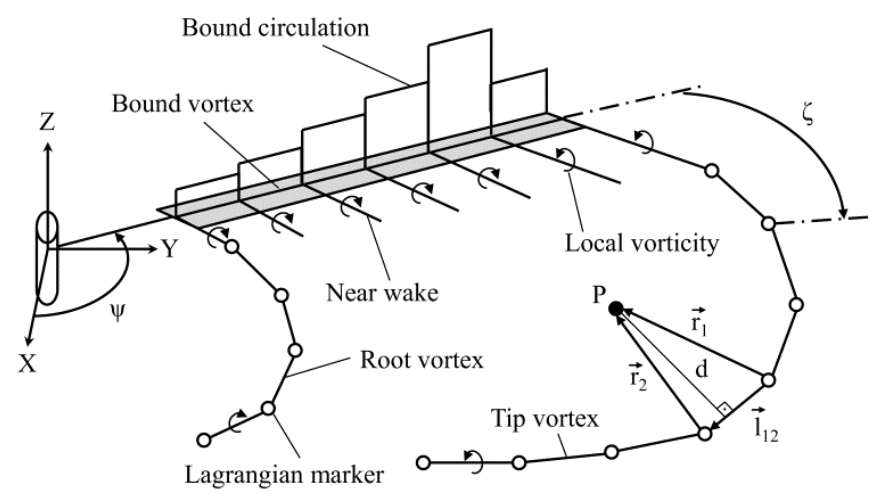

Fig. 2. Free wake model schematic representation.

It is assumed that viscous effects are significant only within the vortex core region of the filaments. Subsequently, the flow can be considered as inviscid outside the vortex singularity [8]. Under this condition, Eq. (1) can be rewritten as:

$\frac{\partial \vec{r}(\psi, \zeta)}{\partial \psi}+\frac{\partial \vec{r}(\psi, \zeta)}{\partial \zeta}=\frac{1}{\Omega} \vec{V}[\vec{r}(\psi, \zeta)]$ 
where $\psi$ is the azimuthal position of the blade, $\zeta$ is the distance along trailed tip vortices, $\vec{r}(\psi, \zeta)$ is the position vector of the Lagrangian marker and $\Omega$ is the rotational speed of the rotor. Eq. (2) is a partial differential equation (PDE) expressed in the blade-fixed coordinate system. The left-hand side is a one dimensional wave equation which is solved by using the pseudo-implicit predictor-corrector, relaxation free-vortex method proposed by Bagai and Leishman [22]. The right-hand side is a highly nonlinear velocity term which includes the flight velocity components and the velocity induced $\vec{V}_{\text {ind }}$ by all the vortex filaments. The velocity induced by a single vortex filament is evaluated through numerical integration using the Biot-Savart law for straight vortex filaments including the Vatistas vortex core model [23]:

$\vec{V}_{\text {ind }}=\frac{\Gamma}{4 \pi}\left(\frac{d}{\left(r_{c}^{2 n}+d^{2 n}\right)^{1 / n}}\right) \frac{\vec{l}_{12}}{\left|\vec{l}_{12}\right|} \cdot\left(\frac{\vec{r}_{1}}{\left|\vec{r}_{1}\right|}-\frac{\vec{r}_{2}}{\left|\vec{r}_{2}\right|}\right) \cdot\left(\frac{\vec{l}_{12} \times \vec{r}}{\left|\vec{l}_{12} \times \vec{r}_{1}\right|}\right)$,

where $\Gamma$ is the circulation strength of the vortex, $\vec{l}_{12}$ is the vortex filament vector, $\vec{r}_{1}$ and $\vec{r}_{2}$ are the position vectors of the arbitrary point $\mathrm{P}$ relative to the Lagrangian markers of the filament, $\vec{r}$ is the position vector of point $\mathrm{P}$ relative to the vortex segment. The perpendicular distance between point $\mathrm{P}$ and vortex filament is $d=\left|\vec{l}_{12} \times \vec{r}\right| /\left|\vec{l}_{12}\right|$. The tangential velocity profile of the Vatistas model is defined by the general constant $n$ which best matches the experimental velocities measured in [23]. Finally, the vortex core radius $r_{c}$ is given by the Squire's equation:

$$
r_{c}(\zeta)=\sqrt{r_{c 0}^{2}+4 \alpha \delta \nu \frac{\zeta}{\Omega}}
$$

where $r_{c 0}$ is the initial core radius, $\alpha=1.25643$ is the Oseen constant and $\delta=1+\alpha_{1} \frac{\Gamma}{v}$ is the eddy viscosity parameter. Bhagwat et al. [24] suggested values in the order of $10^{-3}$ to $10^{-4}$ for the Squire parameter $\alpha_{1}$ to better represent viscous core growth of small scale rotors. Although the initial vortex core radius of $5-10 \%$ chord is typically used for forward flight, a larger initial core radius $r_{c 0}=0.6 c$ has been used in some studies to reduce spurious BVI response in descending flight [25]. Different core radii may be used for the evaluation of induced velocity for wake calculations from those used to compute induced velocity for rotor inflow calculations, to improve convergence stability and speed 
[25]. For the present analysis, the values of $r_{c 0}=0.1 c$ and $\alpha_{1}=0.001$ are used for wake calculations, and $r_{c 0}=0.6 c$ and $\alpha_{1}=0.008$ for rotor inflow calculations. These values provide the best match between predicted and measured wake, airloads and noise data.

The wake is split in the near wake and far wake regions, as depicted in Fig. 2. The near wake is discretized with an azimuthal step of $\Delta \zeta=8^{\circ}$ and truncated at the age of $\zeta=80^{\circ}$. The far wake comprises root and tip vortices, resolving three wake rotations for the calculation of induced flowfield. The near and far wake regions are linked via a vortex roll-up model based on the distribution of bound circulation and local vorticity $\partial \Gamma(r) / \partial r$. The rotor azimuthal step implemented is $\Delta \psi=2^{o}$ to efficiently capture the BVI airloads across the mission. The blades are discretized into 30 radial elements and six vibration modes are considered. The aforementioned resolutions have been selected after sensitivity analyses and literature suggestions [8]. Shed vortices are not resolved in the wake model as they are fundamentally accounted for in the unsteady blade aerodynamic model through the deficiency functions applied on the circulatory normal force coefficient [16].

\subsection{Near-field noise prediction model}

The primary sources of helicopter noise are the main and tail rotors [26]. Rotor noise can be divided into discrete frequency and broadband noise. Discrete frequency noise encompasses the deterministic components of thickness noise, loading noise and high-speed impulsive noise. Thickness noise is due to the displacement of the fluid caused by the blade motion. Loading noise is generated by the forces that act on the fluid because of the pressure distribution around the blade surface. Loading noise usually refers to harmonic loading components. However, impulsive loading components occur as a result of trailed tip vortices interacting with following blades. This is known as BVI noise and is treated separately, due to the specific requirements of the aerodynamic rotor model, as described in section 2.2. High speed impulsive noise occurs at high advancing-tip speeds and is associated with transonic flow effects around the blade. Broadband noise is a result of blade loading associated with turbulent flow in the vicinity of the blade. 
A newly developed FORTRAN 90/95 code is deployed for the prediction of rotor noise across complete four-dimensional operations. This code is an integral implementation of the Ffowcs Williams-Hawkings (FW-H) equation [27], which is the most general form of the Lighthill's acoustic analogy [28]. The FW-H equation is an exact rearrangement of the continuity equation and the Navier-Stokes equations into the form of an inhomogeneous wave equation with monopole and dipole terms across the acoustic data surface and a quadrupole source distribution surrounding it.

$$
\begin{aligned}
\left(\frac{1}{c_{0}^{2}} \frac{\partial^{2}}{\partial t^{2}}-\frac{\partial^{2}}{\partial x_{i}^{2}}\right) p^{\prime}(\vec{x}, t) & =\frac{\partial}{\partial t}\left\{\left[\rho_{0} \vec{v} \cdot \hat{n}+\rho(\vec{u} \cdot \hat{n}-\vec{v} \cdot \hat{n})\right] \delta(f)\right\} \\
& -\frac{\partial}{\partial x_{i}}\left\{\left[\Delta P_{i j} \hat{n}_{j}+\rho u_{i}(\vec{u} \cdot \hat{n}-\vec{v} \cdot \hat{n})\right] \delta(f)\right\}, \\
& +\frac{\partial^{2}}{\partial x_{i} x_{j}}\left[T_{i j} H(f)\right]
\end{aligned}
$$

where $p^{\prime}(\vec{x}, t)$ is the acoustic disturbance evaluated at the observer position $\vec{x}$ and observer time $t$ and $c_{0}$ and $\rho_{0}$ are the speed of sound and density of the quiescent acoustic medium, respectively. Additionally, $\vec{v}$ is the source surface velocity vector, $\vec{u}$ is the fluid velocity vector and $\hat{n}$ is the unit outward normal vector to the source surface. The Lighthill stress tensor is $T_{i j}=\rho u_{i} u_{j}+P_{i j}-c_{0}^{2}\left(\rho-\rho_{0}\right) \delta_{i j}$, with $\rho$ being the local fluid density and $\delta_{i j}$ the Kronecker delta. The compressive stress tensor is defined as $\Delta P_{i j} \hat{n}_{j}=\left(p-p_{0}\right) \delta_{i j}$ after neglecting the viscous forces. The source surface is defined by the function $f=0$ and $H(f)$ is the Heaviside function. The lefthand side term of Eq. (1) represents the three-dimensional wave operator. On the right-hand side there are three source terms, which are monopole, dipole and quadrupole sources, respectively.

Formulation 1A of Farassat [29], [26] is the most extensively utilized and validated integral solution of the FW-H equation. It is based on the free-space Green's function solution of the threedimensional wave equation. Farassat recognized that the wave operator is a linear operator, so both the temporal and spatial derivatives commute [26]. Formulation 1A resolves thickness and loading noise components, which correspond to the monopole and dipole acoustic terms, respectively. The high-speed impulsive noise component which corresponds to the quadrupole term of the FW-H 
equation is neglected. This is valid as long as the rotor tip Mach number is below the transonic limit, which is the typical condition for the majority of civil rotorcraft operations.

Chordwise compact acoustic expressions of the formulation 1A are employed, since the blade chord can be considered small compared to the wavelength of the radiated acoustic content [8]. Brentner et al. [30] introduced a compact form of the loading noise term of the formulation 1A, utilizing the blade-element lift distribution $\vec{L}$ across the blade span. Consequently, the computation reduced from a surface integral to a line integral. For representative values of rotor blade passage frequency (BPF), compact chordwise loading approximation is valid up to the 35th harmonic of the radiated noise spectrum [31]. Lopes [32] presented and validated a compact expression of the monopole term of the formulation $1 \mathrm{~A}$, based on the following geometrically compact assumption: the radiation distance is large compared to the blade chord. The proposed formulation utilizes the spanwise distribution of the airfoil cross-sectional area $A_{c s}$ as a blade design parameter of each blade element with differential span length of $d y$. Hence, the thickness acoustic pressure is also evaluated as a line integral defined by the line equation $f=0$. Using the shorthand notation introduced by Lee et al. [33] and followed by Lopes [32], the compact expressions of thickness and loading acoustic pressure can be formed as follows:

$$
\begin{aligned}
\frac{4 \pi}{\rho_{0}} p_{m}^{\prime}(\vec{x}, t) & =\left.\int_{f=0} A_{c s}\left(W+\frac{\partial W}{\partial \tau}\right) R(2,4)\right|_{r e t} d y \\
& +\left.\int_{f=0} 2 A_{c s} W^{2} R(3,5)\right|_{r e t} d y \\
& -\left.\int_{f=0} c_{0} A_{c s} \frac{\partial \vec{M}}{\partial \tau} \cdot \hat{r} R(2,3)\right|_{r e t} d y \\
4 \pi c_{0} p_{d}^{\prime}(\vec{x}, t)= & \left.\int_{f=0} \frac{\partial \vec{L}}{\partial \tau} \cdot \hat{r} R(1,2)\right|_{r e t} d y \\
& +\left.\int_{f=0} c_{0}(\vec{L} \cdot \hat{r}-\vec{L} \cdot \vec{M}) R(2,2)\right|_{r e t} d y, \\
& +\left.\int_{f=0} \vec{L} \cdot \hat{r} W R(2,3)\right|_{r e t} d y
\end{aligned}
$$


where $p_{m}^{\prime}$ and $p_{d}^{\prime}$ are the monopole and dipole acoustic disturbance terms respectively. The vectors $\vec{L}, \vec{M}$ and $\hat{r}$ correspond to the loading vector, the local relative Mach number vector and the unit radiation vector, respectively. It is noted that the time derivatives are being evaluated in the source time. The subscript "ret" represents numerical integration within the retarded-time (i.e. source-time). The following shorthand functions are being used, as suggested by Lopes [32]:

$$
\begin{aligned}
& R(n, m)=r^{-n}(1-\vec{M} \cdot \hat{r})^{-m}, \\
& W=r \frac{\partial \vec{M}}{\partial \tau} \cdot \hat{r}+c_{0}\left(\vec{M} \cdot \hat{r}-|\vec{M}|^{2}\right) .
\end{aligned}
$$

The source-time-derivative of the function $W$ has been analytically determined by Lee [33]:

$$
\begin{aligned}
\frac{\partial W}{\partial \tau}= & \frac{r^{2} \frac{\partial^{2} \vec{M}}{\partial \tau^{2}} \cdot \hat{r}-3 c_{0} r \frac{\partial \vec{M}}{\partial \tau} \cdot \vec{M}}{r} \\
+ & \frac{c_{0}\left(r \frac{\partial \vec{M}}{\partial \tau} \cdot \hat{r}+c_{0}\left(\left(\frac{\partial \vec{M}}{\partial \tau} \cdot \hat{r}\right)^{2}-|\vec{M}|^{2}\right)\right)}{r}
\end{aligned}
$$

where $r$ is the radiation distance and $|\vec{M}|$ is the magnitude of the local relative Mach number vector.

The overall discrete frequency acoustic pressure $p^{\prime}(\vec{x}, t)$, evaluated at the observer position $\vec{x}$ , located at distance $r$ from the source point and at the observer time $t$ can be expressed as the summary of monopole $p_{m}^{\prime}(\vec{x}, t)$ and dipole $p_{d}^{\prime}(\vec{x}, t)$ acoustic disturbance contributions:

$$
p^{\prime}(\vec{x}, t)=p_{m}^{\prime}(\vec{x}, t)+p_{d}^{\prime}(\vec{x}, t) .
$$

The compact acoustic formulations adopted in this study are compatible with the lifting-linetype aerodynamic input provided by the free-wake airloads model. Moreover, computational savings of up to $99.5 \%$ have been reported with only slight deviations from the corresponding non-compact deterministic acoustic pressure computations [30], [32]. This enables the incorporation of on-the-fly and physics-based rotor source noise prediction into the simulation of complete rotorcraft operations.

Broadband noise is a stochastic noise source; hence turbulence statistics would be required for its first-principles prediction. Furthermore, extensive computational resources would be required 
which goes beyond the purpose of mission-level predictions. The semi-empirical model developed by Pegg [34] is employed for the prediction of broadband noise in the frequency domain. Pegg's model accounts for contributions from boundary layer noise, inflow turbulence noise and vortex noise. It has been shown that the inclusion of Pegg's model accounts for the deficiency in ground noise predictions observed when broadband noise is neglected [35].

\subsection{Far-field noise propagation model}

A source-time dominant algorithm has been developed for the numerical implementation of the retarded-time acoustic formulation, as described in [36]. The source position and time are determined a-priori according to the blade kinematics and flight dynamics calculations. Next, for the requested observer positions, the corresponding observer times are explicitly calculated using the retarded-time equation:

$t=\tau+|\vec{x}-\vec{y}| / c_{0}$,

where $t$ is the observer (i.e. retarded) time, $\tau$ is the source time and $\vec{x}, \vec{y}$ are the observer and the source position vectors, respectively, relative to and expressed in the ground fixed reference frame.

The drawback of this method is that the acoustic pressure time-histories for each observer are unequally spaced in time, thus time-interpolation is needed to resolve acoustic pressure at the desired time intervals. However, co-ordinate transformations between source-observer are evaluated only once per source point, thus significant computational savings occur compared to the traditional observer-time dominant (or retarded-time) numerical algorithm [36].

Fig. 3 presents the summary of the rotor noise model implementation for the prediction of the acoustic impact across the grid of ground observers. A two-step procedure is adopted for the prediction of source noise and its far-field propagation. A fictitious acoustic sphere is considered around the rotorcraft and fixed to the main rotor hub which will represent the source-noise to be propagated to the ground. For each ground observer, a ray-approach is adopted to determine the intersection point between the ray and the sphere. This intersection point serves as an intermediate sphere-observer for the evaluation of acoustic pressure time-history for one rotor revolution. The time-domain acoustic signal is then spectrally analyzed via fast Fourier transformation (FFT) to 
provide a frequency-domain expression of the rotor acoustic impact on the designated sphereobserver. The broadband noise contribution is then added to the deterministic frequency-domain noise components. Formulation 1A does not account for sound attenuation and propagation losses. However this is essential for mission-level applications considering the altitude variations typically encountered by helicopters. The propagation losses during the sound wave travel from the acoustic sphere to the ground observer are accounted for in the frequency domain, assuming no losses within the sphere volume. Atmospheric absorption is assessed using the method proposed by Sutherland et al. [37] and standardized by ISO 9613-1 and ANSI S1.26-1995. The employment of the acoustic sphere aims to the consistent application of far-field propagation from a common geometric starting point for all the individual noise contributions coming from the rotor blades. The sphere has a radius of $150 \mathrm{~m}$, as followed in [10] to properly apply the inverse square law for spherical geometric spreading.

The procedure described above is repeated for the entire set of ground observers. Following this methodology, the evaluation of source noise levels across the entire acoustic sphere surface is avoided, since only the ray-sphere intersection points are considered, as shown in Fig. 3. Consequently, the transition from pre-stored hemisphere databases to the generation of acoustic spheres on-the-fly, across the mission, is realized. Additionally, dedicated acoustic spheres are constructed reflecting the specific flight state of the helicopter. Consequently, the proposed methodology can be considered as generalized and independent of pre-stored databases as input. Moreover, there is no need for interpolation on the non-regular and usually sparse polar grid of conventional noise hemispheres.

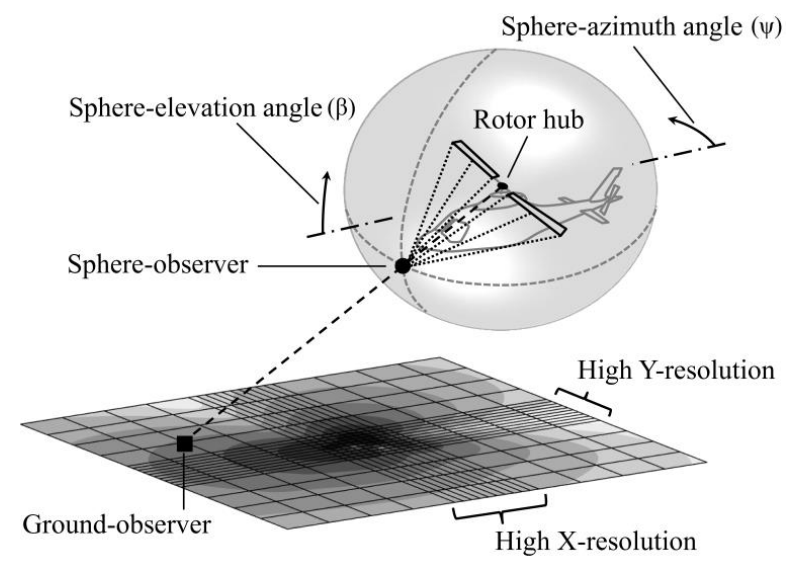

Fig. 3. Rotor noise prediction methodology overview. 
For the purposes of this study, the acoustic content of main rotor has only been included in the analysis. However, the same methodology can be adopted for the prediction of tail rotor noise, or multi-rotor aircraft. Other noise sources such as engine noise or drivetrain noise are of lower intensity and easily attenuated with distance due to their high frequencies. These sources will be neglected as in [6], [9] and [26], although there might be an underprediction of ground noise levels at specific instants of the mission and for observers very close to the helicopter.

\subsection{Integrated numerical procedure}

A three-step integrated procedure has been developed to reduce computational time and enable the generation of acoustic spheres on-the-fly based on high resolution computation of unsteady airloads. This involves the coupling of flight dynamics, elastic blade motion, unsteady airloads and aeroacoustics at mission level. First, the comprehensive code HECTOR is used to compute the flight path, aircraft motion, and engine performance and to update the AUM based on the iterative process described in section 2.1. The converged HECTOR mission output is used to set up the rotor trim requirements for the free-wake airloads model. The airloads model is trimmed for thrust, rolling moment and pitching moment coefficients at each element of the mission for the flight conditions specified by HECTOR. The final unsteady blade airloads (including BVIs), elastic blade motion, as well as blade design are provided by the free-wake airloads model to the aeroacoustics code, as shown in Fig. 4. The rotor airloads prediction procedure described in section 2.3 is executed every time flight conditions change or if the variation of helicopter mass exceeds a user-defined tolerance. In this way, the computationally demanding airloads calculation is only performed when substantial changes in helicopter trim state occur without any significant loss of accuracy. The acoustic sphere generation, as well as, far field noise propagation procedure explained in section 2.4 is executed at each and every trajectory waypoint, as described in the flowchart of Fig. 4. In this context, the noise data calculated on the acoustic sphere surface directly reflects the specific flight state of the rotorcraft, with the sphere observer positions being directly adjusted to each individual ground observer position. 


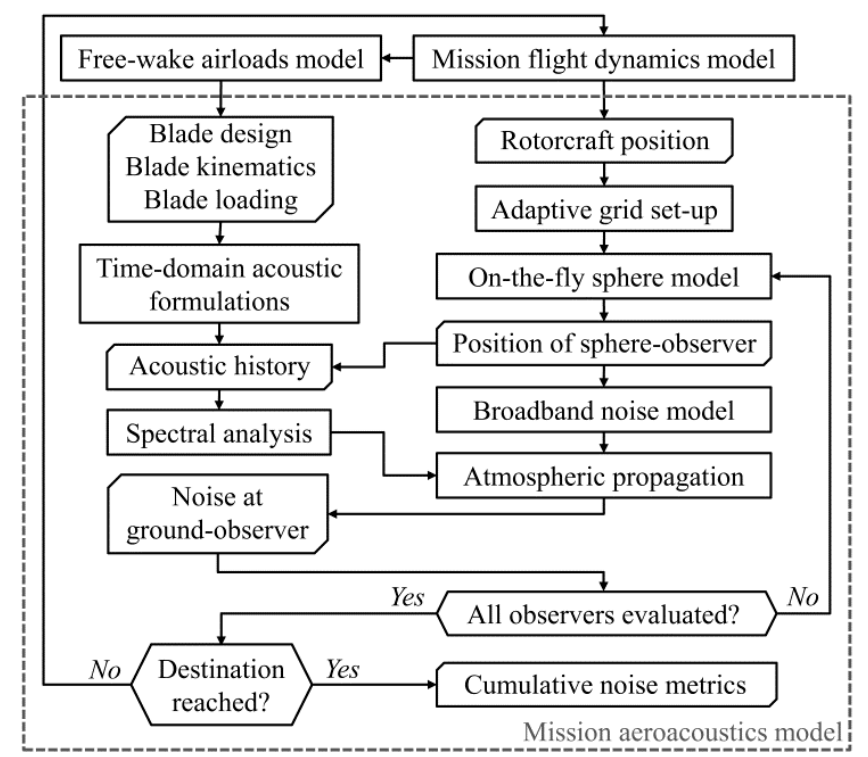

Fig. 4. Numerical procedure for the mission-level noise prediction.

The acoustic sphere generation method proposed in this study becomes beneficial when combined with an appropriate set-up of the ground observer grid. The structure of the ground observer grid determines the accuracy and computational efficiency of the proposed approach at mission level. Regular rotorcraft operations cover hundreds of $\mathrm{km}^{2}$ areas. The complex ground noise patterns require high resolution to adequately capture the associated acoustic phenomena. However, the use of a uniform and dense grid across the entire mission space is prohibitive, due to the limitations in computational time and Random Access Memory (RAM) capabilities of present workstations. Considering that the prominently affected ground regions at each trajectory waypoint are those that lie in the vicinity of the rotorcraft, it can be understood that high grid resolution is only necessary at limited ground regions across the trajectory.

A non-uniform and trajectory-adaptive ground grid is implemented for this study. The entire mission area is covered by a relatively coarse grid. For the area below the rotorcraft, a dense grid is utilized to capture the complex ground noise patterns, as shown in Fig. 3. The position of the densegrid region is continuously updated during the mission, according to the rotorcraft motion. The width as well as the resolution of both coarse and dense-grid regions is determined by the user. An observer grid sensitivity analysis is carried out and analyzed in section 3.2 to form a reliable set of rules for the tuning of the adaptive grid and determination of the employed resolutions. Finally, the free wake code, as well as the aeroacoustics code have been parallelized utilizing the Open Multi-Processing 
(OpenMP) application programming interface [38], which supports shared memory multiprocessing parallel programming. The computational savings of parallelization are reported in section 3.8.

\section{Results and discussion}

\subsection{Comparison with experimental measurements}

The validity of the coupled approach is assessed via the comparison of predicted acoustic pressures and noise footprints with experimental measurements. Fig. 5 represents a comparison of predicted noise with experimental measurements retrieved from [37]. The acoustic pressure time history is predicted for a 4-bladed, $2.9 \mathrm{~m}$ diameter United Technologies freely articulated model rotor of the UH-60A (Black Hawk) helicopter. The exhibited data refers to the microphone 1 measurements which is located in the plane of the rotor and 1.5 rotor diameters upstream of it. The advancing tip Mach number of the rotor is 0.690 . Considering the microphone position and rotor operating state, it becomes clear that the thickness noise component dominates the measurements, although there are slight BVIs which are adequately captured by the model.

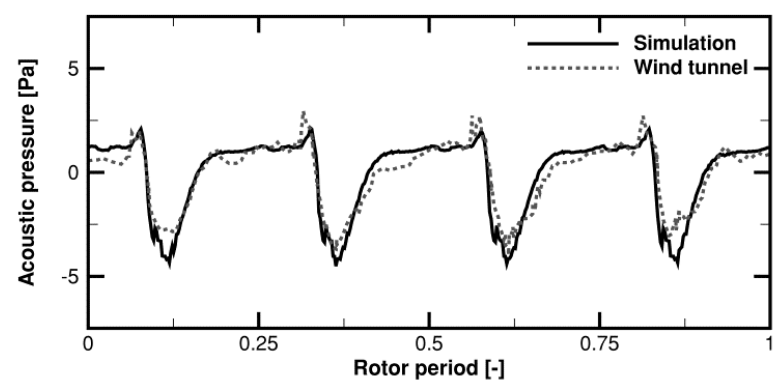

Fig. 5. Acoustic pressure prediction for the UH-60 helicopter model rotor-comparison with wind tunnel data extracted from [39].

The deviation metric adopted for this comparison is the root-mean-square error normalized by the peak-to-peak amplitude (NRMSE), which can be expressed as follows:

$\varepsilon_{N R M S}=\frac{\sqrt{\frac{\sum_{i=1}^{N}\left(p^{\prime}(t)_{\text {meas }}-p^{\prime}(t)_{\text {sim }}\right)^{2}}{N}}}{p^{\prime}(t)_{\text {meas }}^{\text {max }}-p^{\prime}(t)_{\text {meas }}^{\text {min }}}$,

where the subscripts "meas" and "sim" refer to the measured and simulated acoustic pressure, respectively. The superscripts "max" and "min" refer to the maximum and minimum values of 
measured acoustic history, comprising $N$ data points. Good agreement is observed between measured and predicted acoustic pressure, in terms of pulse amplitude and width, resulting in an NRMSE $\varepsilon_{N R M S}=9.31 \%$.

Fig. 6 presents the distribution of the time-derivative of normal force coefficient $d\left(C_{n} M^{2}\right) / d \psi$ across the rotor disc for the HART-II rotor. The rotor is a $40 \%$ scale model of the 4bladed hingeless Bo 105 helicopter rotor. The rotor radius is $\mathrm{R}=2 \mathrm{~m}$ and the rotational speed is $\Omega=109.12 \mathrm{rad} / \mathrm{s}$. The rotor is descending at shaft angle $\alpha_{\mathrm{s}}=-4.5^{\circ}$ with advance ratio $\mu=0.15$ and trust coefficient $\mathrm{C}_{\mathrm{T}}=0.0044$. The markers represent the locations with blade-vortex miss distance smaller than the blade chord [21]. It can be observed that the predicted BVI locations coincide with the peaks of the derivative of normal force coefficient; hence BVIs are properly captured by the airloads model.

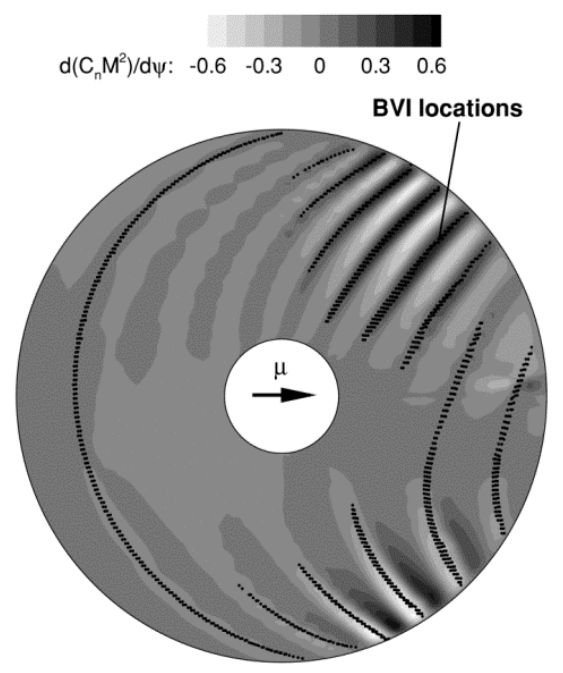

Fig. 6. Distribution of the derivative of normal force coefficient and BVI locations for the HART-II rotor.

Fig. 7 presents a comparison of predicted noise with experimental measurements undertaken within the HART-II experimental campaign [40] conducted in the DNW German-Dutch wind tunnel. The rotor characteristics and operating conditions are same as described in Fig. 6. The microphone is $2.3 \mathrm{~m}$ below the rotor disc, at a distance of $0.0 \mathrm{~m}$ from the rotor hub in the upstream direction and of $1.81 \mathrm{~m}$ on the advancing side. It can be observed that the acoustic pressure signal is governed by 
strong BVIs due to the negative rotor disc incidence with respect to the free stream. The predicted results are in good agreement with the flight measurements with NRMSE $\varepsilon_{\text {NRMS }}=8.96 \%$.

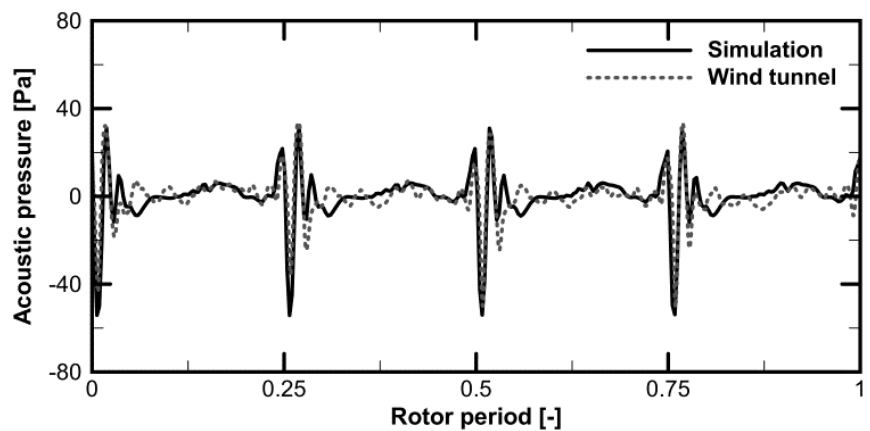

Fig. 7. Acoustic pressure prediction for the HART-II rotor-comparison with experimental data extracted from [40].

Fig. 8 (b) presents the Overall Sound Pressure Level (OASPL) contours for the same experiment [40], measured across a horizontal plane $2.3 \mathrm{~m}$ below the rotor disc. Acoustic frequencies between the $6^{\text {th }}$ and the $40^{\text {th }}$ BPF harmonic have only been considered in the frequency spectrum integration. Two distinct lobes of high noise levels, primarily due to advancing and retreating side BVIs are formed. Similar patterns have also been observed in [41]. The simulation noise levels presented in Fig. 8 (a) are slightly overpredicted at the advancing side BVI. Generally, good agreement is observed between the measured and predicted noise levels with NRMSE $\varepsilon_{\text {NRMS }}=12.86 \%$ for the entire contour map.

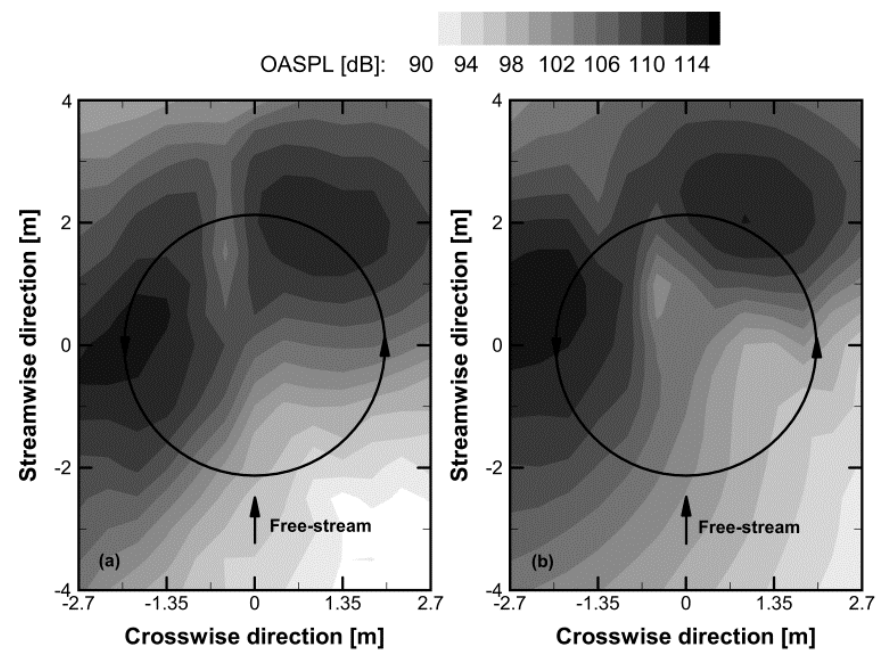

Fig. 8. Ground noise footprint for the HART-II rotor-comparison with experimental data extracted from [40]: (a) wind tunnel; (b) simulation. 


\subsection{Observer grid sensitivity analysis}

The adaptive grid implemented in this study includes a high-resolution region, fixed to the helicopter position and continuously updated during the mission to capture the complex noise patterns at the predominantly affected ground regions. A lower resolution is selected for the rest of the mission area, as illustrated in Fig. 3, to reduce the required computational resources. The four operating parameters that predominantly affect the acoustic footprint patterns are: the flight altitude $h$, the rotor advance ratio $\mu$, the flightpath angle $\gamma$ and the rotor thrust coefficient $C_{T}$, as these are directly related to the radiation distance, source velocity, source noise directivity and blade loading, respectively.

\section{Table 1.}

Baseline design parameters for the MBB Bo 105 helicopter main rotor

\begin{tabular}{cc}
\hline Parameter & Value \\
\hline Blade count & 4 \\
Rotor radius [m] & 4.91 \\
Root cut-out & 0.21 \\
Rotor solidity & 0.07 \\
Blade twist $\left[^{\circ}\right.$ ] & -8 \\
Blade chord [m] & 0.27 \\
Rotational speed [ $\mathbf{r a d} / \mathbf{s}]$ & 44.4 \\
\hline
\end{tabular}

A sensitivity analysis is carried out to determine the width as well as the number of observers in the high-resolution grid region. The helicopter employed for this study is the MBB Bo 105 helicopter. It is a TEL multipurpose helicopter, featuring a 4-bladed hingeless rotor. An extensive validation of performance and flight dynamics of this model is provided in [13]. Table 1 summarizes the main design characteristics of the main rotor of the selected helicopter. The acoustic footprints are 
assessed for a wide range of the four aforementioned governing parameters, as described in Table 2 .

The ground Cartesian coordinates, as well as flight altitude are normalized with the rotor radius R.
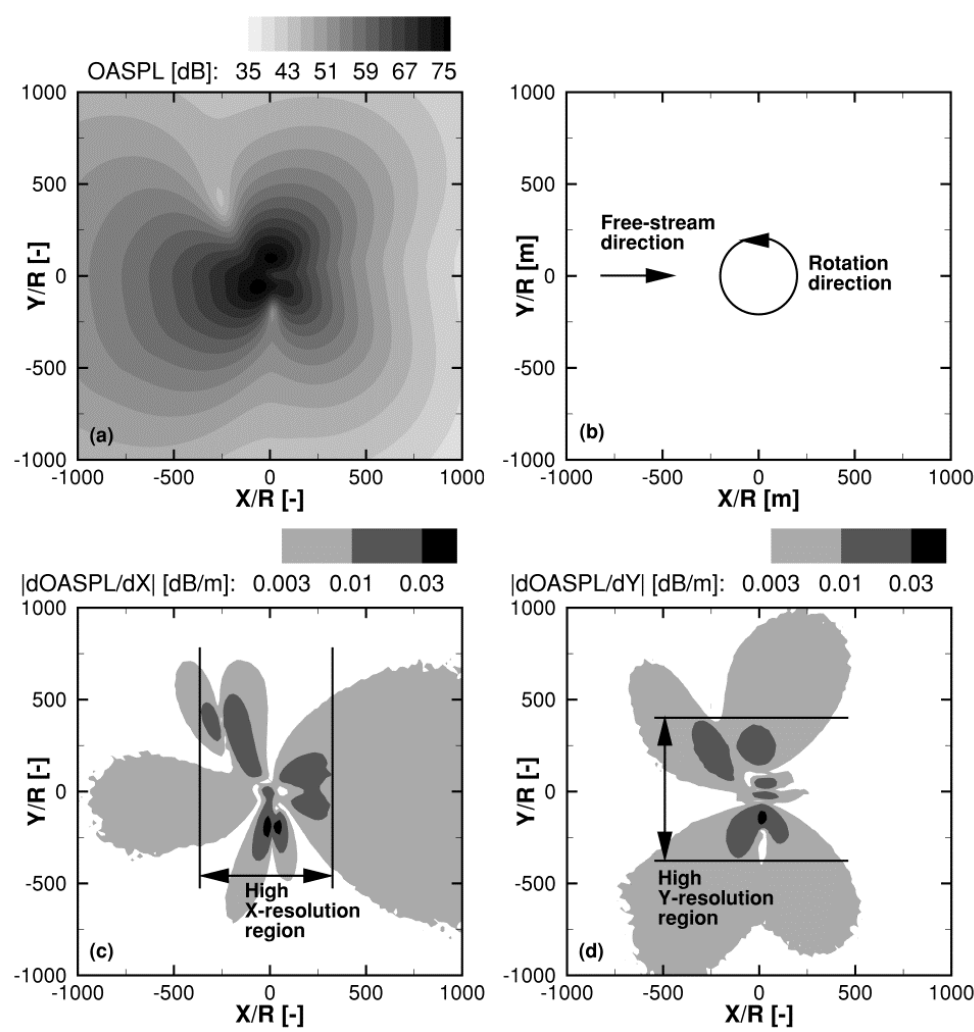

Fig. 9. Observer grid sensitivity analysis in forward flight: (a) Ground noise footprint for $\mathrm{h} / \mathrm{R}=100$, $\mu=0.16, C_{T}=0.0044$; (b) Free-stream and rotation directions; (c) OASPL absolute X-gradient; (d) OASPL absolute Y-gradient.

Five different values (with corresponding IDs) have been determined for each governing parameter, which leads to 20 total cases examined. At each ID, only the values of the governing parameter of interest are varying keeping the rest three parameters at their default values annotated in brackets in Table 2. For example, when examining the impact of advance ratio $\mu$ the ID=2 corresponds to $\mu=0.08$, with the rest parameters at default $\mathrm{h} / \mathrm{R}=100, \gamma=0 \mathrm{deg}$. and $\mathrm{C}_{\mathrm{T}}=0.0044$. Subsequently, the X and Y Cartesian gradients of the OASPL recorded are calculated to determine the sensitivity of noise patterns in the aforementioned directions. A fourth-order central finite difference scheme has been employed for the estimation of OASPL gradients.

Fig. 9 (a) illustrates the ground footprint for the MBB Bo 105 helicopter, cruising at normalized altitude $h / R=500$, with advance ratio $\mu=0.16$ and thrust coefficient $C_{T}=0.0044$. The helicopter is located at $\mathrm{X} / \mathrm{R}=0 \mathrm{~m}, \mathrm{Y} / \mathrm{R}=0 \mathrm{~m}$ and the free-stream direction is towards the positive side 
of X-axis, as shown in Fig. 9 (b). The noise metric adopted in this study is the Overall Sound Pressure Level (OASPL), which is calculated by integrating over the resolved frequencies of the acoustic signal [42]. Thickness noise has in-plane directivity so ground noise is generally higher upstream of the helicopter. Loading noise has mainly out-of-plane directivity, which in combination with the higher Mach number values of the advancing side, results in higher loading noise below the right side of the helicopter.

Table 2.

Cases examined in the observer grid sensitivity analysis.

\begin{tabular}{cc}
\hline Parameter & Examined values (IDs 1 to 5) \\
\hline $\mathbf{h} / \mathbf{R}[-]$ & $5,50,(100), 250,400$ \\
$\boldsymbol{\mu}[-]$ & $0.00,0.08,(0.16), 0.24,0.32$ \\
$\gamma\left[^{0}\right]$ & $-7,-3,(0), 3,7$ \\
$\mathbf{C}_{\mathrm{T}}[-]$ & $0.0030,0.0038,(0.0044), 0.0050,0.0058$
\end{tabular}

Fig. 9 (c) and (d) depict the absolute value of the $\mathrm{X}$ and $\mathrm{Y}$ gradients utilizing a three-level logarithmic scale, from 0.003 to $0.03 \mathrm{~dB} / \mathrm{m}$. Gradients greater than $0.01 \mathrm{~dB} / \mathrm{m}$ are observed in ground region extending from $\mathrm{X} / \mathrm{R}=-325$ to $\mathrm{X} / \mathrm{R}=286$ in the $\mathrm{X}$-direction, and from $\mathrm{Y} / \mathrm{R}=-320$ to $\mathrm{Y} / \mathrm{R}=411$ in the Y-direction. Limited regions with gradients greater than $0.03 \mathrm{~dB} / \mathrm{m}$ are located inside the aforementioned area. The rest of the mission area is described by OASPL gradients lower than 0.003 $\mathrm{dB} / \mathrm{m}$. Similar patterns are observed for the rest of the examined cases, albeit with variations in the location and width of the high-gradient regions.

Fig. 10 summarizes the results of observer grid sensitivity analysis providing the extent of the high-gradient regions in both $\mathrm{X}$ and $\mathrm{Y}$ positive/negative directions. Each of the governing operational parameters previously defined is represented by one colored bar. The IDs 1 to 5 refer to the five different values examined for each governing parameter. At each ID, only the values of the governing parameter of interest are varying; the rest three governing parameters are assigned their default value indicated in Table 2. It can be concluded that the $\mathrm{X} / \mathrm{R}=-500$ to $\mathrm{X} / \mathrm{R}=500$ and the $\mathrm{Y} / \mathrm{R}=-500$ to 
$\mathrm{Y} / \mathrm{R}=500$ ranges adequately determine the limits of the high-resolution grid region across the entire envelope of operating conditions. The observer spacing in both Cartesian directions inside the highresolution area will be $\mathrm{dX}=\mathrm{dY}=30 \mathrm{~m}$, which will lead to a numerical accuracy of approximately $1 \mathrm{~dB}$ based on the maximum value recorded for the OASPL gradient. A spacing of $d X=d Y=180 \mathrm{~m}$ will be adopted outside of the high-resolution area where OASPL gradients are significantly lower. As discussed in section 2.5 , the high-resolution region of the ground grid dynamically adjusts with mission time by "following" the helicopter across its trajectory.

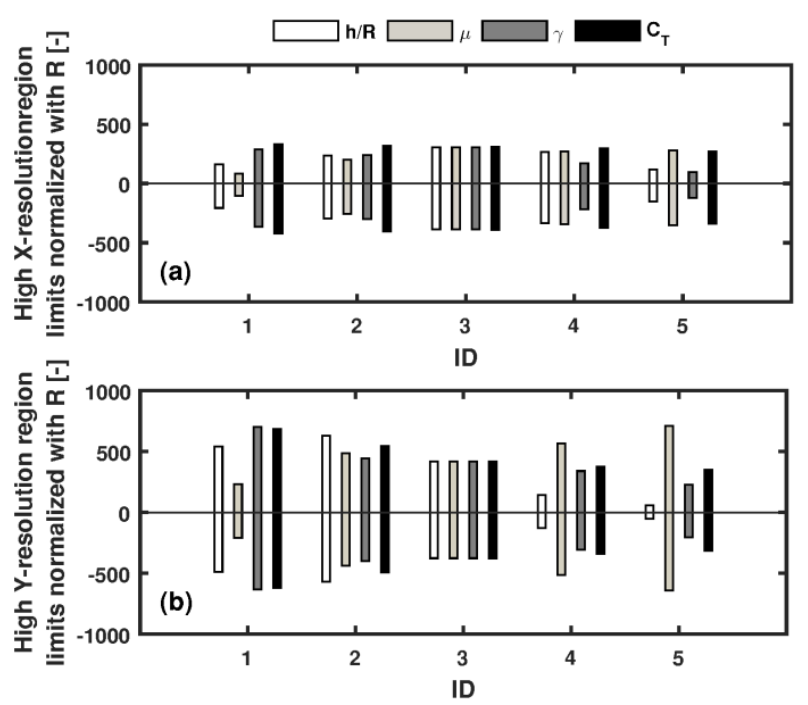

Fig. 10. Summary of the limits of the high-resolution regions, exported from the observer grid sensitivity analysis: (a) X-direction; (b) Y-direction.

\subsection{Mission definition}

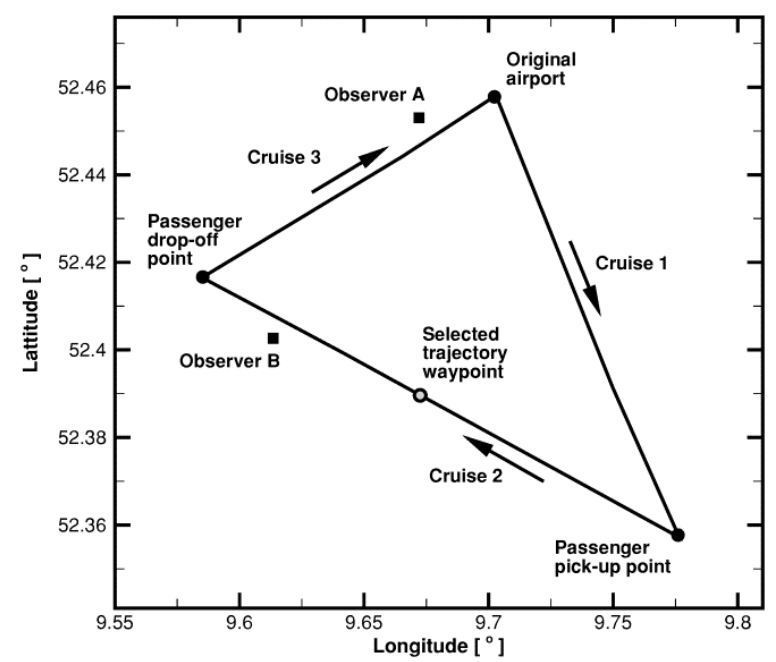

Fig. 11. PAT scenario geographical definition in WGS 84 . 
An integrated methodology has been established for the prediction of source and ground noise, which now allows the application on realistic rotorcraft operations. Two generic, fourdimensional missions representative of modern TEL helicopter operations have been defined: a passenger air-transport mission (PAT) and a search and rescue (SAR) mission. The incorporated operational procedures in terms of geographical location selection, deployed airspeed, altitude, climb/descent rates, and idle times have been defined in collaboration with the European Helicopter Operators Committee (EHOC). It is noted that the effect of change in air density and speed of sound is inherently accounted in the variation of flight altitude. The geographical representation in terms of global coordinates, are illustrated in Fig. 11 and Fig. 12 for the PAT and SAR case, respectively. Individual trajectory waypoints are selected for both scenarios (grey-filled circle markers) to investigate the radiation-sphere and instantaneous ground footprints. Two isolated observers (A and B) are defined for each mission to assess the perceived noise time-variations at representative ground locations.

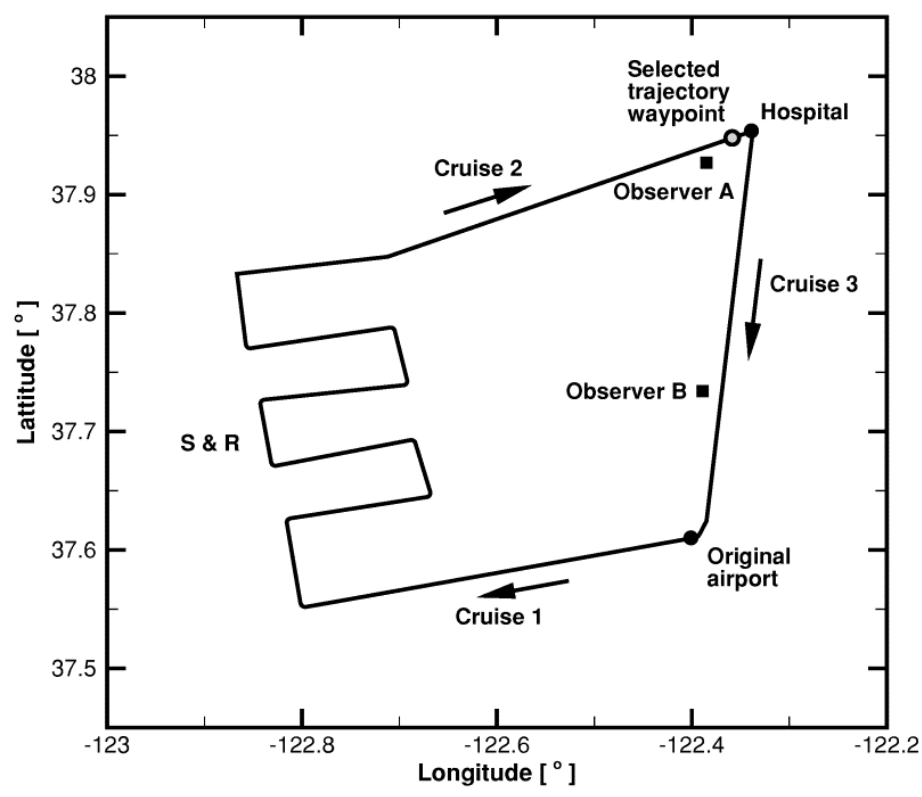

Fig. 12. SAR scenario geographical definition in WGS 84. 

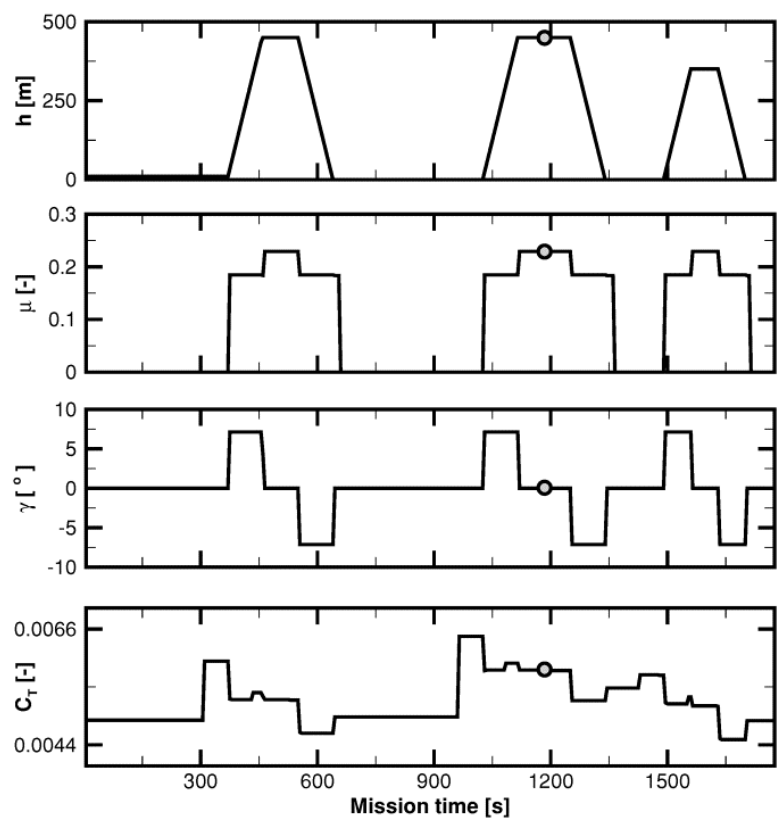

Fig. 13. PAT scenario time variations of deployed operational altitude advance ratio, flightpath angle and thrust coefficient.

The PAT mission assumes that the rotorcraft takes-off from Hanover Airport (Germany) and cruises in order to pick up passengers from a designated location. It subsequently cruises towards the Garbsen Hotel to drop them off and then returns back to Hanover Airport. The SAR mission assumes that the helicopter takes off from San Francisco Airport (USA) and then travels towards the Gulf of the Farallones. The helicopter is then assumed to engage in a search pattern to trace and pick up citizens in distress from an assumed naval incident location. After successfully locating the citizens in distress, the helicopter SAR crew picks them up while hovering over the imaginary incident zone. The citizens are then transported to the San Pablo Hospital, where the helicopter drops them off and subsequently transits back to San Francisco Airport. 

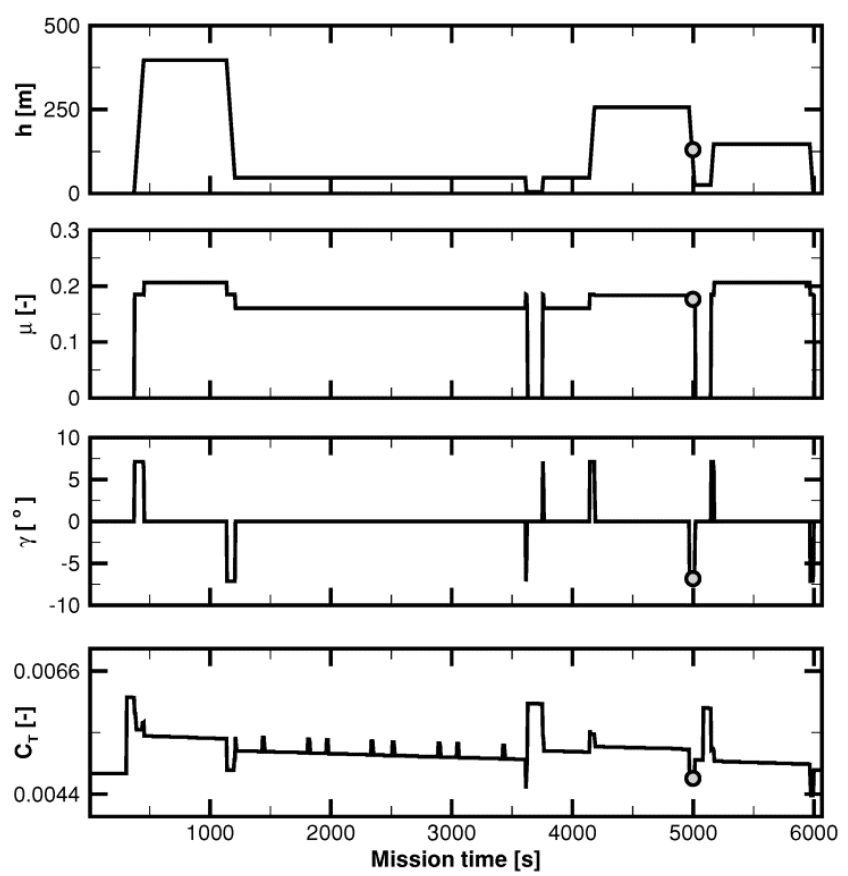

Fig. 14. SAR scenario time variations of deployed operational altitude, advance ratio, flightpath angle and thrust coefficient.

Climb and descent rates are held fixed at $5 \mathrm{~m} / \mathrm{s}$. A time step of $5 \mathrm{~s}$ is used for each individual mission segment. During idle operation, the overall helicopter power requirements are assumed to be equal to $10 \%$ of maximum contingency engine shaft power. The acoustic impact of the helicopter in idle is neglected.

The time variations of the helicopter operating parameters are represented in Fig. 13 and Fig. 14, for the PAT and SAR case, respectively. The PAT mission comprises three legs in total and covers a total area of $323 \mathrm{~km}^{2}$. The cruise speed within the first two legs is $50 \mathrm{~m} / \mathrm{s}$ at an altitude of $\mathrm{h}=450 \mathrm{~m}$, while for the final leg, the cruise speed is $45 \mathrm{~m} / \mathrm{s}$ at an altitude of $\mathrm{h}=350 \mathrm{~m}$. The total PAT mission duration is 0.49 hours, extending to a total range of 36.22 kilometers and consuming $55.19 \mathrm{~kg}$ of fuel. As regards the SAR mission, a total area of $3969 \mathrm{~km}^{2}$ is examined. The helicopter spends almost half of the mission time engaged in the SAR pattern, where the deployed flight speed and altitude are $35 \mathrm{~m} / \mathrm{s}$ and $50 \mathrm{~m}$, respectively. The total SAR mission duration is 1.68 hours, extending to a total range of 208.45 kilometers and consuming $191.41 \mathrm{~kg}$ of fuel. 


\subsection{Impact of fuel burn on rotor noise generation}

The amount of fuel burned during the SAR mission equals approximately to $8.7 \%$ of the takeoff mass of the helicopter. The AUM reduction due to fuel burn has direct impact on the $\mathrm{C}_{\mathrm{T}}$ time history, where a $9.26 \%$ reduction is observed between the beginning of Cruise 1 and end of Cruise 3 sections of the SAR mission. $\mathrm{C}_{\mathrm{T}}$ is an integral non-dimensional metric of rotor disc loading; hence its gradual reduction within the mission course due to fuel burn is essentially reflected on the corresponding loading noise acoustic spheres, as depicted in Fig. 15.

A two-dimensional representation of the acoustic sphere is adopted in the context of this work, in a way that the three-dimensional spherical surface is "un-wrapped" on a two-dimensional map. The center of the acoustic sphere is fixed to the aircraft hub, as illustrated in Fig. 3. The azimuth angle is measured from the tail following the direction of rotation of the main rotor. The elevation angle is measured from the horizontal plane attached to the rotor hub and is positive upwards. Only the lower noise hemisphere is presented since the upper does not radiate noise to the ground.

Differences of up to $+/-5 \mathrm{~dB}$ are present on the acoustic spheres between the beginning and the ending of the mission. It is noted that both $\mu$ and $\gamma$ are the same between the compared mission time-instants. The relative deviations in the rotor trim angles and blade flapping angles are less than $3 \%$, leading to negligible directivity variations between the compared states; hence the dominant factor that changes between the two trim states is $\mathrm{C}_{\mathrm{T}}$. The reduction of $\mathrm{C}_{\mathrm{T}}$ is attributed to the reduction of fuselage AUM which affects the vertical force requirement for the main rotor. This results in a more negative tip-path-plane angle, increased downwash and reduced angles of attack at the aft region of the rotor disc. This leads to a re-distribution of the elementary thrust across the rotor disc by increasing the loading at the front and significantly reducing at the aft region of the rotor disc. Consequently, the OASPL of the advancing side of the noise hemisphere is reduced, whilst it increases at the retreating side. It becomes clear that the impact of fuel burn is crucial on the prediction of helicopter $\mathrm{AUM}, \mathrm{C}_{\mathrm{T}}$ and finally, ground noise, especially for long-range missions. 

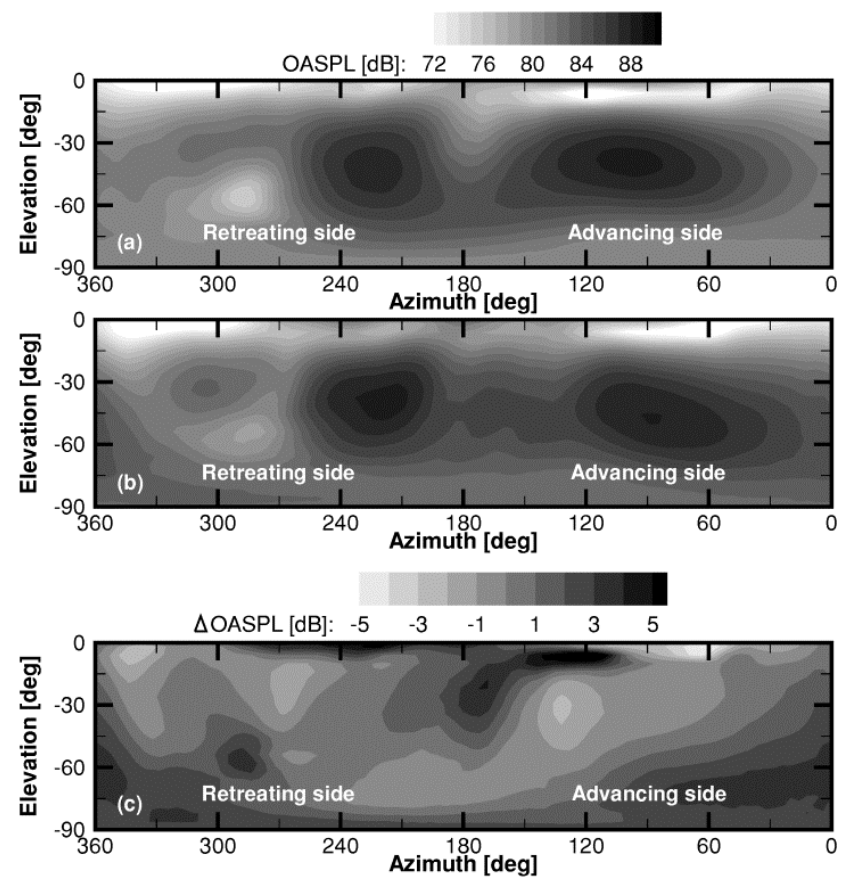

Fig. 15. Loading noise acoustic spheres for the SAR scenario: (a) beginning of cruise $1: t=455 \mathrm{~s}, \mu=$ 0.206, $\gamma=0^{\circ}, \mathrm{C}_{\mathrm{T}}=0.0054$; (b) ending of the cruise 3: $\mathrm{t}=5965 \mathrm{~s}, \mu=0.206, \gamma=0^{\circ}, \mathrm{C}_{\mathrm{T}}=0.0049$; (c) OASPL difference between (a) and (b).

\subsection{Noise footprint for individual trajectory waypoints}

Fig. 16 and Fig. 17 demonstrate the acoustic spheres (a) and the corresponding ground footprints (b) for the selected trajectory waypoints of the PAT and SAR case, respectively. The high resolution regions in the $\mathrm{X}$ and $\mathrm{Y}$ direction are also superimposed. Considering the adopted ground grid set-up as described in section 3.2, a resolution of $30 \mathrm{~m}$ is adopted in the high-gradient region and $180 \mathrm{~m}$ in the rest parts of the ground grid in both Cartesian directions. As a result, a total of 56,538 observers occur for the PAT case, resulting in an $84.24 \%$ reduction compared to the uniform observer grid of $30 \mathrm{~m}$ resolution. For the SAR scenario, a total of 238,252 observers have been utilized, resulting in a $94.59 \%$ reduction in the number of employed observers, relative to the uniform grid configuration. It is noted that the high-resolution region follows the motion of the helicopter; hence a new computational grid is set up at each trajectory waypoint to capture the complex ground noise pattern with the least computational effort. 

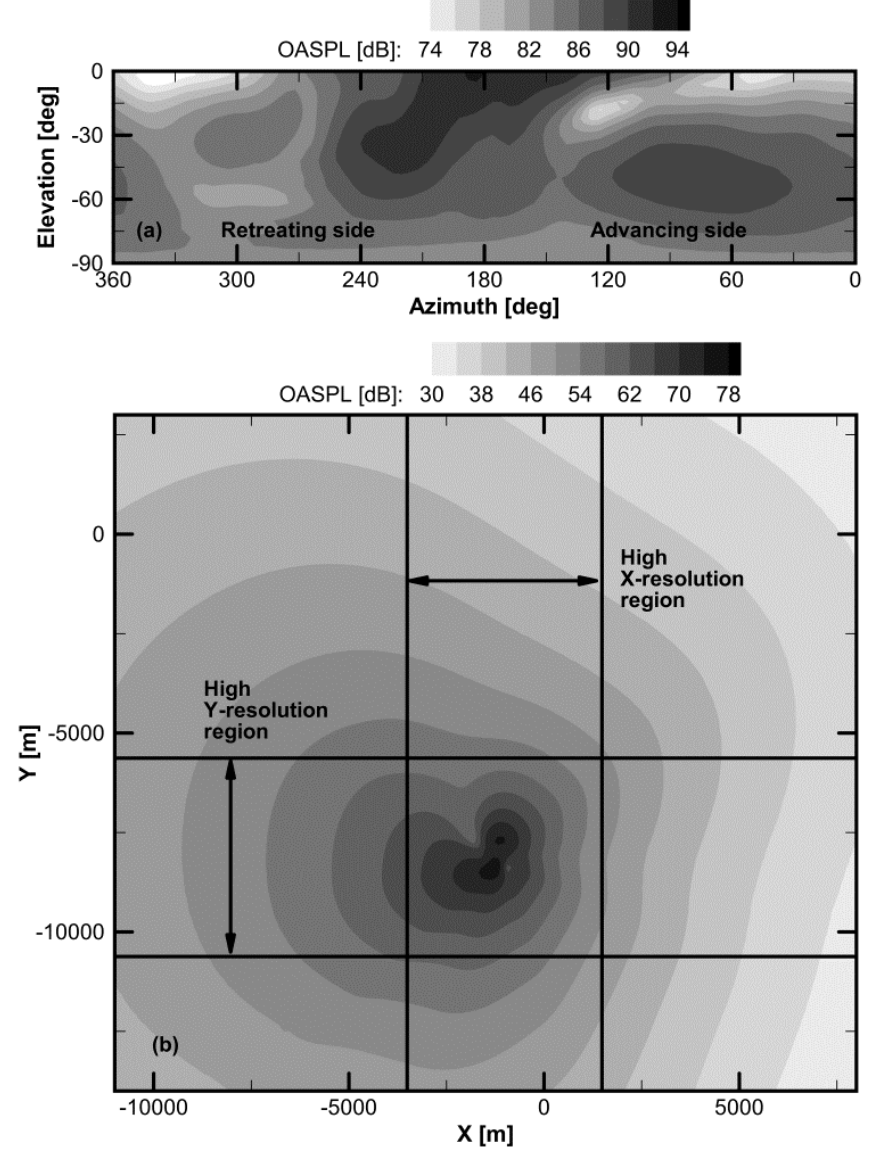

Fig. 16. Selected trajectory waypoint of the PAT scenario: (a) acoustic sphere; (b) corresponding ground noise footprint: $\mathrm{t}=1180 \mathrm{~s}, \mu=0.230, \gamma=0^{\circ}, \mathrm{C}_{\mathrm{T}}=0.0060$.

For the PAT mission, the selected trajectory waypoint corresponds to cruise conditions, at an advance ratio $\mu=0.23$ and altitude $\mathrm{h}=450 \mathrm{~m}$. Thickness noise is dominant in the plane and upstream of the rotor, thus a strong peak is observed at $\psi=180^{\circ}$ and $\beta=0^{\circ}$ on the acoustic sphere. Significant loading noise is observed at $\psi=90^{\circ}$ and $\beta=-45^{\circ}$ generated by the aft loaded region of the rotor. For the SAR case a time-instant at altitude $\mathrm{h}=132 \mathrm{~m}$ with the helicopter in descent with $\gamma=-7.13^{\circ}$ at advance ratio $\mu=0.18$ is selected. The BVIs generated due to negative disc incidence angle leads to increased out-of-plane loading noise, especially below the advancing side at $\psi=90^{\circ}$, $\beta=-60^{\circ}$.

The aforementioned source-noise patterns are reflected on the ground footprint contours. Increased noise levels are observed upstream of the helicopter in the PAT selected waypoint, whilst 
significant noise radiation is observed below the advancing side in the SAR selected snapshot as shown in the magnified area of Fig. 17 (b). It can also be observed that the affected ground regions extend to kilometer-range distances. The quantification of the corresponding noise exposure times becomes of utmost priority for the mission-level assessment of the acoustic impact on the ground.

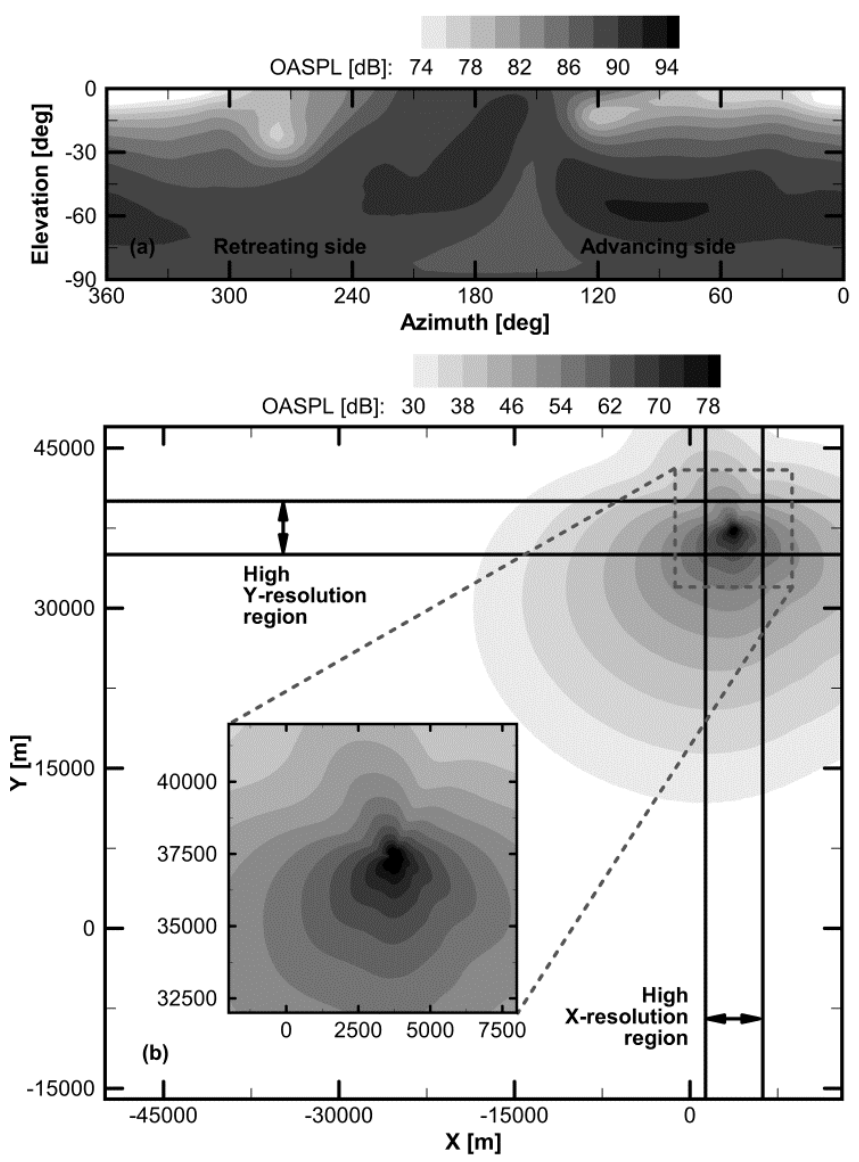

Fig. 17. Selected trajectory waypoint of the SAR scenario: (a) acoustic sphere; (b) corresponding ground noise footprint: $\mathrm{t}=4990 \mathrm{~s}, \mu=0.185, \gamma=-7.13^{\circ}, \mathrm{C}_{\mathrm{T}}=0.0047$.

\subsection{Acoustic time histories for isolated ground observers}

Fig. 18 presents the observer-time distributions of noise levels for two isolated observers (A and B) for the PAT (a) and the SAR (b) mission. The observer time is calculated using Eq. (12) with the aircraft-time (mission-time) serving as source time. The noise travel-time is estimated assuming uniform atmosphere and utilizing the radiation distance between the rotor hub and the observer. Negligible rotor noise radiation is assumed during idle periods. For the PAT scenario, generally 
higher noise levels are observed, because of the shorter range, which keeps the helicopter close to the observers during the entire mission. Additionally, the cruise advance ratio is higher compared to the SAR mission; hence the higher thickness noise levels contribute significantly to the overall noise.
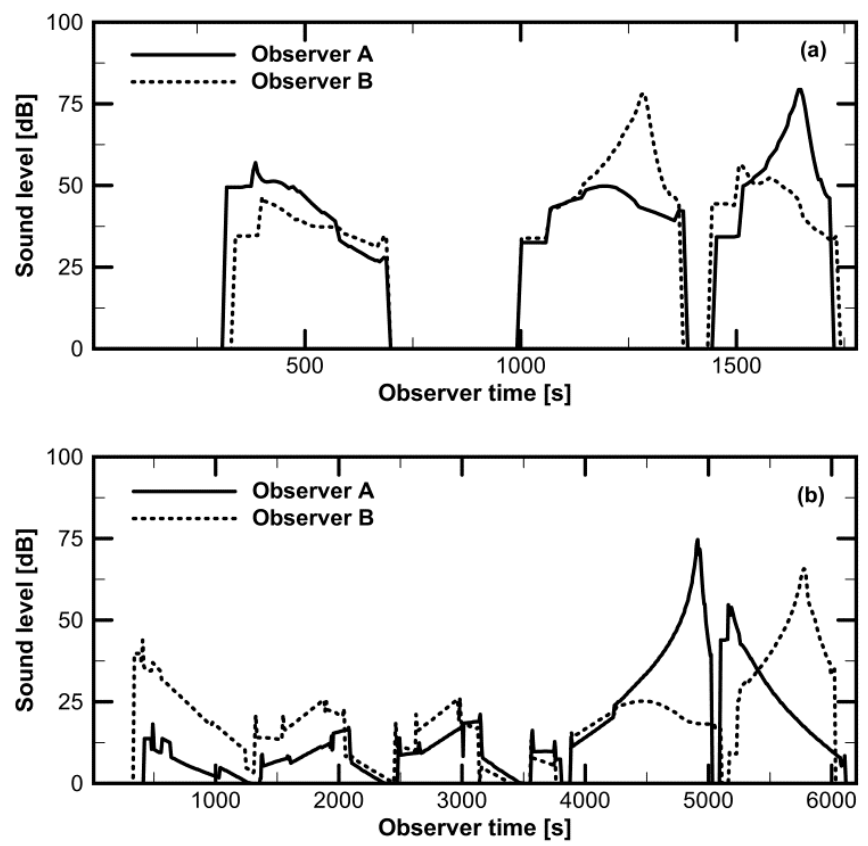

Fig. 18. Acoustic histories for selected isolated observers A and B for each mission: (a) PAT; (b) SAR.

The highest noise levels are recorded when the radiation distance is minimized. However, there is an extended time-period before and after the peaks, where gradually increasing and decreasing, respectively, noise levels are recorded. Some short-duration peaks are present in the SAR case for both the examined observers, which are due to the banked turns which take place during the search and rescue mission task element. The change of orientation results in slight peaks in the $\mathrm{C}_{\mathrm{T}}$ time history as seen in Fig. 14, which contribute to increased loading noise. Moreover, significant thickness noise radiation towards the observers $\mathrm{A}$ and $\mathrm{B}$ occurs during the orientation changes across the SAR mission task elements. The relative time-exposure is of importance for the holistic missionlevel noise assessment. This indicates that a cumulative metric has to be adopted, that apart from absolute noise levels, it also accounts for noise exposure times. 


\subsection{Cumulative metrics of mission acoustic impact}
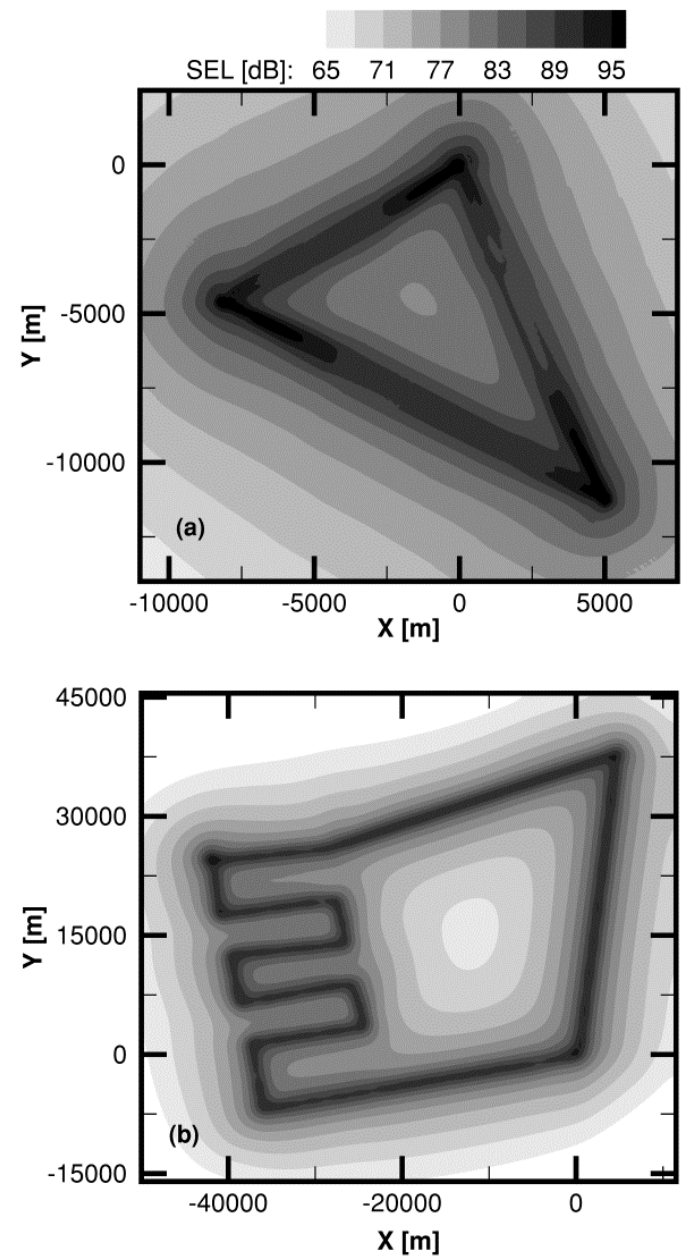

Fig. 19. Sound exposure levels: (a) PAT scenario; (b) SAR scenario.

In sections 3.5, 3.6 it was shown that rotorcraft missions can affect wide ground regions and for extended periods of time. Cumulative metrics are essential for the inclusion of both spatial and temporal effects into the mission acoustic impact assessment. Fig. 19 illustrates the sound exposure levels (SEL) for the PAT and SAR missions, respectively. SEL is defined as the energy averaged sound level over a specified period of time or single noise event, with reference duration of 1 second [42]:

$S E L=10 \log _{10}\left[\frac{1}{T_{0}} \int_{t_{1}}^{t_{2}} 10 \frac{\operatorname{OASPL}(t)}{10} d t\right]$, 
where $T_{0}$ is the reference duration of $1 \mathrm{sec}$ and $t_{2}-t_{1}$ is the actual duration of the acoustic event. In the context of this study, the SEL is calculated applying continuous time integration for the entire exposure period at each observer position, as followed in [1]. The PAT scenario has generally higher overall noise levels due to the higher cruise speeds compared to the SAR mission.

It is observed that the ground regions located before the landing points experience the highest SEL. That is due to temporal annoyance effects accounted for in the calculation of the SEL. The inplane directed thickness noise is focused on the ground upstream of the helicopter during trimmed descent; hence it has a continuous contribution in the upstream ground regions, up until the landing points. Additionally, as shown in Fig. 16, significant BVI noise contributions are added to the overall annoyance during descent, which also have temporal contribution to the SEL.

\subsection{Assessment of computational performance}

The computational performance of the individual models is assessed to quantify the computational savings introduced by the parallelization and the implementation of trajectory adaptive ground observer grids. The free-wake rotor airloads code and the rotor aeroacoustics code are assessed separately for individual trim states, although the exported outcomes can be extended to mission level. The assessment has been undertaken on a personal computer equipped with 16 gigabytes (GB) of RAM and a central processing unit (CPU) with 4 cores ( 8 threads) operating at 3.60 $\mathrm{GHz}$.

\section{Table 3.}

Rotor airloads model computational performance.

\begin{tabular}{cccc}
\hline No of & \multicolumn{3}{c}{ CPU time [mins] } \\
threads & $\boldsymbol{\Delta} \boldsymbol{\psi}=\mathbf{1}^{\mathbf{0}}$ & $\boldsymbol{\Delta} \boldsymbol{\psi}=\mathbf{2}^{\mathbf{0}}$ & $\boldsymbol{\Delta} \boldsymbol{\psi}=\mathbf{5}^{\mathbf{0}}$ \\
\hline $\mathbf{1}$ thread & 167.2 & 44.5 & 23.8 \\
$\mathbf{8}$ threads & 29.5 & 7.1 & 4.3 \\
\hline
\end{tabular}


Table 3 presents the elapsed computational times for the free-wake rotor airloads code to trim the rotor for the HART-II case presented in section 3.1. Computational savings of up to $84.05 \%$ are recorded between the single and the 8-threaded execution for the $\Delta \psi=2^{\circ}$ azimuthal resolution case. The rest of wake characteristics have been specified in section 2.2.

Table 4 presents a comparison of elapsed computational times for the aeroacoustics module of the framework referring to one single trim point of the PAT mission scenario. The trim point under investigation is the selected trajectory waypoint of the PAT case presented in section 3.5. Computational savings of up to $76.00 \%$ are recorded between the single and the 8 -threaded execution for the $\Delta \psi=2^{\circ}$ azimuthal resolution case with adaptive ground grids implemented. The employed number of observers is 56,538 , as specified in section 3.5. It can be seen that the use of the proposed grid adaptation method results in a $62.15 \%$ reduction in computing time requirements. This allows the method to be used on-the-fly without the need of pre-stored noise data.

\section{Table 4.}

Rotor aeroacoustics model computational performance.

\begin{tabular}{cccc}
\hline No of threads/Grid structure & \multicolumn{3}{c}{ CPU time [mins] } \\
& $\boldsymbol{\Delta} \boldsymbol{\psi}=\mathbf{1}^{\mathbf{0}}$ & $\mathbf{\Delta} \boldsymbol{\psi}=\mathbf{2}^{\mathbf{0}}$ & $\boldsymbol{\Delta} \boldsymbol{\psi}=\mathbf{5}^{\mathbf{0}}$ \\
\hline $\mathbf{1}$ thread/Uniform grid & 448.3 & 222.7 & 91.5 \\
$\mathbf{8}$ threads/Uniform grid & 112.4 & 56.8 & 22.3 \\
$\mathbf{1}$ thread/Adaptive grid & 180.1 & 89.6 & 36.2 \\
$\mathbf{8}$ threads/Adaptive grid & 45.8 & 21.5 & 8.1 \\
\hline
\end{tabular}

\section{Conclusions}

An analytical approach has been developed for the prediction of the acoustic impact of fourdimensional rotorcraft operations. It comprises a mission analysis comprehensive code, an aero-elastic high resolution airloads free wake model and a newly developed rotor aeroacoustics model. Good 
agreement has been shown between predicted and measured acoustic pressures and noise footprint contours. A procedure for the generation of acoustic spheres on-the-fly, coupled with trajectoryadaptive ground observer grids has been incorporated into a mission-level simulation environment. The developed methodology has been successfully applied towards the assessment of ground noise impact of two generic missions of a TEL helicopter model, representative of the MBB Bo 105.

The on-the-fly acoustic sphere generation method, which employs computationally efficient mission flight dynamics coupled with high-resolution aero-elastic free wake computations, has been the enabler for the mission-level application of noise prediction. When combined with trajectoryadaptive ground grids, reductions of up to $94.59 \%$ have been calculated in the total of employed ground observers, relative to uniform grid configurations for a long range mission. The devised methodology has been successful in handling long-range and long-duration rotorcraft missions. The impact of fuel burn across the mission has been proven crucial for the long-duration missions. A $9.26 \%$ reduction in the value of main rotor thrust coefficient has been observed between the beginning and the ending of the mission, which corresponds to differences of up to $+/-5 \mathrm{~dB}$ on the source loading noise radiation.

The analytical approach described in this paper enables the implementation of physics-based rotor noise prediction in four-dimensional mission simulations, keeping the associated computational overhead at acceptable levels. The proposed strategy constitutes a generalized methodology that is independent of pre-stored acoustic data. It is offered in a form that is readily available for application on any specified rotorcraft model and on any designated four-dimensional operation.

\section{Conflict of interest statement}

None declared.

\section{Acknowledgments}

The authors would like to acknowledge Dr. Alejandro Castillo Pardo for his advice and technical contribution. 


\section{References}

[1] D. A. Conner and J. A. Page, "A Tool for Low Noise Procedures Design and Community Noise Impact Assessment: The Rotorcraft Noise Model (RNM)," in AHS International Meeting on Advanced Rotorcraft Technology and Life Saving Activities, Heli Japan, Japan, 2002.

[2] M. Gervais and R. Heger, "Validation of EC130 and EC135 Environmental Impact Assessment Using HELENA," in American Helicopter Society 66th Annual Forum, Phoenix, $A Z, 2010$.

[3] I. Goulos, V. Pachidis, R. D’Ippolito, J. Stevens, and C. Smith, “An Integrated Approach for the Multidisciplinary Design of Optimum Rotorcraft Operations," J. Eng. Gas Turbines Power, vol. 134, no. 9, p. 091701, 2012.

[4] F. H. Schmitz and G. Gopalan, "Quasi-Static Flight Path Control of Helicopter Blade Vortex Interaction Noise in a Steady Wind,” J. Am. Helicopter Soc., vol. 52, no. 1, pp. 15-23, 2007.

[5] G. Gopalan, F. H. Schmitz, C. Park, and B. W. Sim, "Flight Path Management and Control Methodology to Reduce Helicopter Blade-Vortex Interaction (BVI) Noise," in American Helicopter Society Vertical Lift Aircraft Design Conference, San Francisco, CA, 2000.

[6] E. Greenwood, R. Rau, B. May, and C. Hobbs, "A Maneuvering Flight Noise Model for Helicopter Mission Planning,” Am. Helicopter Soc. 71st Annu. Forum, 2015.

[7] H. Chen, K. S. Brentner, L. V. Lopes, and J. F. Horn, “A Study of Rotorcraft Noise Prediction in Maneuvering Flight," in 42nd AIAA Aerospace Sciences Meeting and Exhibit, Reno, NV, 2004, no. 2003.

[8] H. Chen, K. S. Brentner, S. Ananthan, and J. G. Leishman, "A Computational Study of Helicopter Rotor Wakes and Noise Generated During Transient Maneuvers," J. Am. Helicopter Soc., vol. 53, no. 1, pp. 37-55, 2008.

[9] H. Yin, J., \& Buchholz, “Toward Noise Abatement Flight Procedure Design: DLR Rotorcraft Noise Ground Footprints Model,” J. Am. Helicopter Soc., vol. 52, no. 2, pp. 90-98, 2007.

[10] M. Gennaretti, J. Serafini, G. Bernardini, A. Castorrini, G. De Matteis, and G. Avanzini, "Numerical Characterization of Helicopter Noise Hemispheres," Aerosp. Sci. Technol., vol. 
52, pp. 18-28, 2016.

[11] M. Gennaretti, G. Bernardini, J. Serafini, A. Anobile, and S. Hartjes, "Helicopter Noise Footprint Prediction in Unsteady Maneuvers," Int. J. Aeroacoustics, vol. 16, no. 3, pp. 165$180,2017$.

[12] I. Goulos, P. Giannakakis, V. Pachidis, and P. Pilidis, "Mission Performance Simulation of Integrated Helicopter-Engine Systems Using an Aeroelastic Rotor Model," J. Eng. Gas Turbines Power, vol. 135, no. 9, p. 091201, 2013.

[13] I. Goulos, V. Pachidis, and P. Pilidis, "Helicopter Rotor Blade Flexibility Simulation for Aeroelasticity and Flight Dynamics Applications," J. Am. Helicopter Soc., vol. 59, no. 4, pp. $1-18,2014$.

[14] I. Goulos, V. Pachidis, and P. Pilidis, "Lagrangian Formulation for the Rapid Estimation of Helicopter Rotor Blade Vibration Characteristics,” Aeronaut. J., vol. 118, no. 1206, pp. 861901,2014

[15] I. Goulos, "An Improved Analytical Approach for Modeling the Effect of Rotor Wake Curvature Using Finite-State Induced Flow Models," J. Am. Helicopter Soc., vol. 61, no. 3, pp. $1-16,2016$.

[16] J. G. Leishman and T. S. Beddoes, "A Semi-Empirical Model for Dynamic Stall," J. Am. Helicopter Soc., vol. 3, pp. 3-17, 1989.

[17] D. A. Peters, D. Doug Boyd, and C. Jian He, "Finite-State Induced-Flow Model for Rotors in Hover and Forward Flight," J. Am. Helicopter Soc., vol. 34, no. 4, pp. 5-17, 1974.

[18] EUROCONTROL and IfEN, "WGS 84 Implementation Manual,” Brussels, Belgium. 1998.

[19] W. L. MacMillan, "Development of a Modular-Type Computer Program for the Calculation of Gas Turbine Off-Design Performance," Ph.D. thesis, Cranf. Univ. Cranfield, Bedfordshire, $U K, 1974$.

[20] A. Castillo Pardo, I. Goulos, and V. Pachidis, "Modelling and analysis of coupled flap-lagtorsion vibration characteristics helicopter rotor blades," Proc. Inst. Mech. Eng. Part G J. Aerosp. Eng., vol. 231, no. 10, pp. 1804-1823, 2016.

[21] A. Castillo Pardo, "Aeroelastic Simulation of Rotorcraft Propulsion Systems," Ph.D. thesis, 
Cranf. Univ. Cranfield, Bedfordshire, UK, 2017.

[22] A. Bagai and J. G. Leishman, "Rotor Free-Wake Modeling Using a Pseudo-Implicit Technique — Including Comparisons with Experimental Data," J. Am. Helicopter Soc., vol. 40, no. 3, pp. $29-41,1995$.

[23] G. H. Vatistas, V. Kozel, and W. C. Mih, "A simpler model for concentrated vortices," Exp. Fluids, vol. 11, no. 1, pp. 73-76, 1991.

[24] M. J. Bhagwat and J. G. Leishman, "Generalized viscous vortex model for application to freevortex wake and aeroacoustic calculations," in American Helicopter Society 58th Annual Forum and Technology Display, Montreal, Canada, 2002.

[25] B. G. van der Wall, J. W. Lim, J. D. Baeder, and D. D. Boyd, "The HART II international workshop: an assessment of the state-of-the-art in comprehensive code prediction," CEAS Aeronaut. J., vol. 4, no. 3, pp. 223-252, 2013.

[26] K. S. Brentner and F. Farassat, "Modeling aerodynamically generated sound of helicopter rotors," Prog. Aerosp. Sci., vol. 39, no. 2-3, pp. 83-120, 2003.

[27] J. E. Ffowcs Williams and D. L. Hawkings, "Sound Generation by Turbulence and Surfaces in Arbitrary Motion," Philos. Trans. R. Soc. London. Ser. A, Math. Phys. Sci., vol. 264, no. 1151, pp. 321-342, 1969.

[28] M. J. Lighthill, “On Sound Generated Aerodynamically. I. General Theory,” Proceedings of the Royal Society A: Mathematical, Physical and Engineering Sciences, vol. 211, no. 1107. pp. $564-587,1952$.

[29] F. Farassat and G. P. Succi, "The prediction of helicopter rotor discrete frequency noise," in American Helicopter Society 38th Annual Forum, Washington, DC, 1982.

[30] K. S. Brentner, C. L. Burley, and M. A. Marcolini, "Sensitivity of Acoustic Predictions to Variation of Input Parameters,” J. Am. Helicopter Soc., vol. 39, no. 3, pp. 43-52, 1994.

[31] C. C. Hennes, H. Chen, and K. S. Brentner, "Influence of Transient Flight Maneuvers on Rotor Wake Dynamics and Noise Radiation," in AHS 4th Decennial Specialist's Conference on Aeromechanics, San Francisco, CA, 2004.

[32] L. V. Lopes, "Compact Assumption Applied to the Monopole Term of Farassat' s 
Formulations," 21st AIAA/CEAS Aeroacoustics Conf. AIAA 2015-2673, pp. 1-22, 2015.

[33] S. Lee, K. S. Brentner, F. Farassat, and P. J. Morris, "Analytic Formulation and Numerical Implementation of an Acoustic Pressure Gradient Prediction," J. Sound Vib., vol. 319, no. 3-5, pp. 1200-1221, 2009.

[34] R. Pegg, "A Summary and Evaluation of Semi-Empirical Methods for the Prediction of Helicopter Rotor Noise,” NASA TM, vol. 80200, 1979.

[35] R. Snider, T. Samuels, B. Goldman, and K. Brentner, "Full-Scale Rotorcraft Broadband Noise Prediction and its Relevance to Civil Noise Certification Criteria," 69th Annu. AHS Forum, Phoenix, AZ, 2013.

[36] B. A. Goldman and K. S. Brentner, "New Algorithms for Reduced Memory and Real-Time Noise Prediction," in 18th AIAA/CEAS Aeroacoustics Conference, Colorado Springs, CO, 2012.

[37] H. E. Bass, L. C. Sutherland, A. J. Zuckerwar, D. T. Blackstock, and D. M. Hester, "Atmospheric absorption of sound : Further developments," J. Acoust. Soc. Am., vol. 97, no. 1, pp. 680-683, 1995.

[38] L. Dagum and R. Menon, "OpenMP: An Industry-Standard API for Shared-Memory Programming," IEEE Comput. Sci. Eng., vol. 5, no. 1, pp. 46-55, 1998.

[39] J. A. Visintainer, M. A. Marcolini, C. L. Burley, and S. R. Liu, "Acoustic Predictions Using Measured Pressures from a Model Rotor in the DNW," J. Am. Helicopter Soc., vol. 38, no. 3, pp. 35-44, 1993.

[40] B. G. van der Wall, C. L. Burley, Y. Yu, H. Richard, K. Pengel, and P. Beaumier, "The HART II test - Mesurement of helicopter rotor wakes," Aerosp. Sci. Technol., vol. 8, no. 4, pp. 273284,2004

[41] M. Gennaretti, G. Bernardini, J. Serafini, and G. Romani, "Rotorcraft comprehensive code assessment for blade - vortex interaction conditions," Aerosp. Sci. Technol., vol. 80, pp. 232246, 2018.

[42] R. L. Bennett and K. S. Pearsons, "Handbook of Aircraft Noise Metrics," NASA Contract. Rep. $3406,1981$. 
2019-04-03

\section{Integrated methodology for the prediction of helicopter rotor noise at mission level}

\section{Vouros, Stavros}

\section{Elsevier}

Vouros S, Goulos I, Pachidis V. (2019) Integrated methodology for the prediction of helicopter rotor noise at mission level. Aerospace Science and Technology, Volume 89, June 2019, pp. 136-149 https://doi.org/10.1016/j.ast.2019.03.061

Downloaded from Cranfield Library Services E-Repository 